\title{
Chemically tagging the Hyades stream: does it partly originate from the Hyades cluster?*
}

L. Pompéia, ${ }^{1,2}$ T. Masseron, ${ }^{1}$ B. Famaey,${ }^{3,4}$ S. Van Eck, ${ }^{1}$ A. Jorissen,${ }^{1} \dagger$ I. Minchev,${ }^{5}$ A. Siebert, ${ }^{3}$ C. Sneden, ${ }^{6}$ J. R. D. Lépine, ${ }^{7}$ C. Siopis,${ }^{1}$ G. Gentile, ${ }^{1,8}$ T. Dermine, ${ }^{1}$ E. Pasquato, ${ }^{1}$ H. Van Winckel, ${ }^{9}$ C. Waelkens, ${ }^{9}$ G. Raskin, ${ }^{9}$ S. Prins,${ }^{9}$ W. Pessemier, ${ }^{9}$ H. Hensberge, ${ }^{10}$ Y. Frémat, ${ }^{10}$ L. Dumortier ${ }^{10}$ and O. Bienaymé ${ }^{3}$

${ }^{1}$ Institut d'Astronomie et d'Astrophysique, Université Libre de Bruxelles, CP 226 Bruxelles, Belgium

${ }^{2}$ Universidade do Vale do Paraíba, Av. Shishima Hifumi, 2911, São José dos Campos, 12244-000 SP, Brazil

${ }^{3}$ Observatoire Astronomique, Université de Strasbourg, CNRS UMR 7550, 67000 Strasbourg, France

${ }^{4}$ AIfA, Universtät Bonn, 53121 Bonn, Germany

${ }^{5}$ AIP, An der Sterwarte 16, 14482 Potsdam, Germany

${ }^{6}$ Department of Astronomy and McDonald Observatory, The University of Texas, Austin, TX 78712, USA

${ }^{7}$ Universidade de São Paulo, IAG, C.P. 3386, 01060-970 São Paulo, Brazil

${ }^{8}$ Sterrenkundig Observatorium, Universiteit Gent, Krijgslaan 281, 9000 Gent, Belgium

${ }^{9}$ Instituut voor Sterrekunde, Katholiek Universiteit Leuven, Celestijnenlaan 200D, 3001 Leuven, Belgium

${ }^{10}$ Royal Observatory of Belgium, Avenue Circulaire 3, 1180 Bruxelles, Belgium

Accepted 2011 March 9. Received 2011 March 9; in original form 2011 January 13

\begin{abstract}
The Hyades stream has long been thought to be a dispersed vestige of the Hyades cluster. However, recent analyses of the parallax distribution, of the mass function, and of the actionspace distribution of stream stars have shown it to be rather composed of orbits trapped at a resonance of a density disturbance. This resonant scenario should leave a clearly different signature in the element abundances of stream stars than the dispersed cluster scenario, since the Hyades cluster is chemically homogeneous. Here, we study the metallicity as well as the element abundances of $\mathrm{Li}, \mathrm{Na}, \mathrm{Mg}, \mathrm{Fe}, \mathrm{Zr}, \mathrm{Ba}, \mathrm{La}, \mathrm{Ce}, \mathrm{Nd}$ and $\mathrm{Eu}$ for a random sample of stars belonging to the Hyades stream, and compare them with those of stars from the Hyades cluster. From this analysis: (i) we independently confirm that the Hyades stream cannot be solely composed of stars originating in the Hyades cluster; (ii) we show that some stars (namely 2/21) from the Hyades stream nevertheless have abundances compatible with an origin in the cluster; (iii) we emphasize that the use of $\mathrm{Li}$ as a chemical tag of the cluster origin of mainsequence stars is very efficient in the range $5500 \mathrm{~K} \leq T_{\text {eff }} \leq 6200 \mathrm{~K}$, since the Li sequence in the Hyades cluster is very tight, while at the same time spanning a large abundance range; (iv) we show that, while this evaporated population has a metallicity excess of $\sim 0.2$ dex with respect to the local thin-disc population, identical to that of the Hyades cluster, the remainder of the Hyades stream population has still a metallicity excess of $\sim 0.06-0.15$ dex, consistent with an origin in the inner Galaxy and (v) we show that the Hyades stream can be interpreted as an inner 4:1 resonance of the spiral pattern: this then also reproduces an orbital family compatible with the Sirius stream, and places the origin of the Hyades stream up to $1 \mathrm{kpc}$ inwards from the solar radius, which might explain the observed metallicity excess of the stream population.
\end{abstract}

Key words: Galaxy: evolution - Galaxy: kinematics and dynamics - open clusters and associations: individual: Hyades.

${ }^{\star}$ Based on observations obtained with the HERMES/Mercator spectrograph/telescope installed at the Roque de los Muchachos Observatory, La Palma, Spain.

†E-mail: alain.jorissen@ulb.ac.be

\section{INTRODUCTION}

It has been known for a long time that a spatially unbound group of stars in the solar neighbourhood is sharing the same kinematics 
as the Hyades open cluster (e.g. Eggen 1958). Assuming that it is a vestige of an initially more massive Hyades cluster which dispersed with time, with the result that its distribution function is still presently evolving towards equilibrium and does not yet satisfy the Jeans theorem, Eggen called this kinematically cold group the Hyades supercluster. More generally, it is called the Hyades moving group or Hyades stream, since Eggen's hypothesis that kinematic groups of this type are indeed such cluster remnants has been largely debated for many years. Actually, such groups may also be generated by a number of global dynamical mechanisms. Most of the mechanisms able to generate such unbound groups of stars moving in a peculiar fashion are linked with the non-axisymmetry of the Galaxy, namely with the presence of a rotating central bar (e.g. Dehnen 1998, Fux 2001, Minchev et al. 2010) and of spiral arms (e.g. Quillen \& Minchev 2005; Antoja et al. 2009) or both (see Quillen 2003; Minchev \& Famaey 2010).

A way to discriminate between these two hypotheses is the "chemical tagging' of stars belonging to the moving groups (Freeman \& Bland-Hawthorn 2002; De Silva, Freeman \& Bland-Hawthorn 2009). With this technique, a true Eggen moving group (or true supercluster) was recently tracked down, namely the HR1614 moving group (Eggen 1978; Feltzing \& Holmberg 2000), in which the high level of chemical homogeneity is supporting the case that it is a relic of an ancient star-forming event (De Silva et al. 2007). Conversely, the Hercules stream was found to be composed of stars whose abundance pattern matches that of the disc stars (Bensby et al. 2007), which could be interpreted as being associated with the outer Lindblad resonance of the bar (Dehnen 1998, Fux 2001).

Here, we intend to analyse the abundance trends of a sample of stars belonging to the Hyades stream, in order to check whether it is compatible with Eggen's scenario, with the resonant scenario, or with a mix of both. Indeed, the evaporation of open clusters and the dynamical perturbations linked with density perturbers are not mutually incompatible phenomena. Stars originating from a 600Myr old cluster had time to disperse over more than $500 \mathrm{pc}$ in the disc (e.g. Bland-Hawthorn, Krumholz \& Freeman 2010), but have retained the same guiding radius and same tangential velocity $V$ (Woolley 1961). This does not prevent an overdensity in velocity space due to orbits trapped at resonance to overlap with this cluster remnant.

The study of the kinematics of $\mathrm{K}$ and $\mathrm{M}$ giant stars in the solar neighbourhood, combining CORAVEL radial velocities (Baranne, Mayor \& Poncet 1979) with Hipparcos parallaxes and proper motions (Famaey et al. 2005), already indicated that the Hyades stream could not be solely composed of coeval stars evaporated from the primordial Hyades cluster, because the Hertzsprung-Russell diagram was very similar for stars in the stream and in the field, indicating a wide range of ages for stars belonging to the stream. This was later confirmed by a detailed analysis of the distribution of stars in parallax space as compared to the parallax corresponding to the Hyades cluster's 600-Myr isochrone (Famaey, Siebert \& Jorissen 2008). Bovy \& Hogg (2010) recently reached the same conclusion. Moreover, at about the same time, radial velocities, masses and metallicities of more than $14000 \mathrm{~F}$ and $\mathrm{G}$ dwarfs stars were also published as the Geneva-Copenhagen (GC) Survey (Nordström et al. 2004; Holmberg, Nordström \& Andersen 2007; Holmberg et al. 2009), supplementing the Hipparcos parallaxes and proper motions. The distribution of these main-sequence stars in velocity space confirmed that the Hyades stream was the most prominent feature on top of the background velocity ellipsoid (Fig. 1). Sellwood (2010) then estimated the actions of individual stars from their observed coordinates and velocities, and examined the stellar

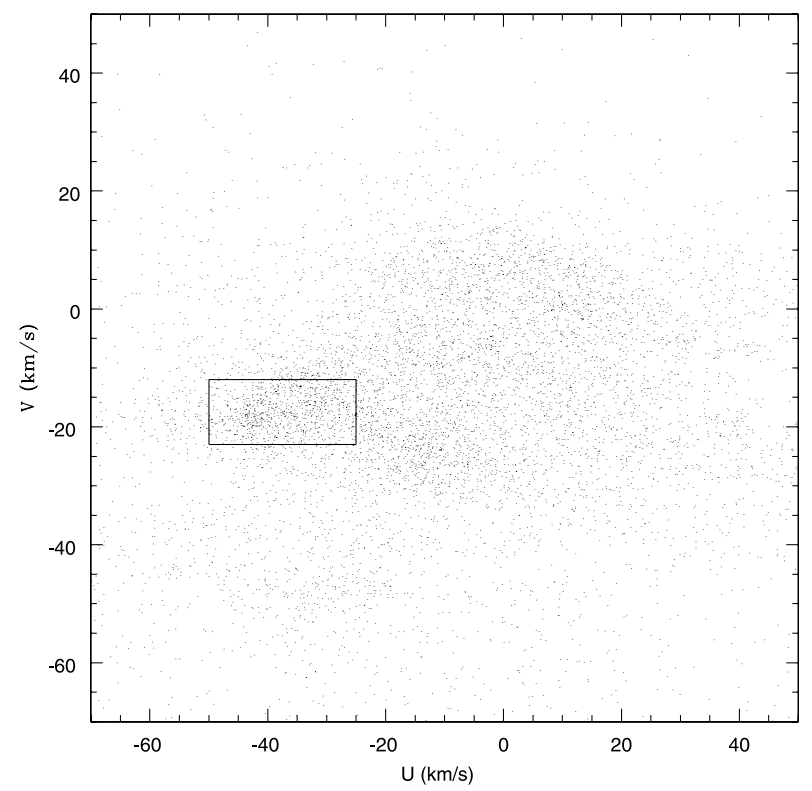

Figure 1. The full Geneva-Copenhagen (GC) sample from Holmberg, Nordström \& Andersen (2009) in the $U-V$ plane. The Hyades box is also indicated at $-23 \mathrm{~km} \mathrm{~s}^{-1} \leq V \leq-12 \mathrm{~km} \mathrm{~s}^{-1}$ and $-50 \mathrm{~km} \mathrm{~s}^{-1} \leq U \leq$ $-25 \mathrm{~km} \mathrm{~s}^{-1}$.

distribution in the $J_{\mathrm{R}}-J_{\phi}$ action space. He found about 5 per cent of the GC stars concentrated along a resonance line in action space, which was interpreted as a signature of scattering at the inner Lindblad resonance of a spiral pattern. ${ }^{1}$ This overdensity in action space precisely corresponds to the Hyades stream in velocity space, in accordance with the model of Quillen \& Minchev (2005). What is more, Famaey et al. (2007) compared the mass distribution of stars belonging to the Hyades stream with the initial mass function of the Hyades cluster and with the present-day mass function of the galactic disc, showing that it was in disagreement with the former and in agreement with the latter, thus also favouring a dynamical, resonant origin for the stream. However, the latter analysis was compatible with a proportion of at least 15 per cent of stream stars actually being past members of the Hyades cluster. It was also found that, while thin-disc stars have a mean metallicity $[\mathrm{Fe} / \mathrm{H}] \simeq-0.15$, the mean metallicity of stars moving with the Hyades (among which only $\sim 25$ per cent of field stars are from the background velocity ellipsoid, the rest constituting the Hyades overdensity in velocity space) was $[\mathrm{Fe} / \mathrm{H}]=-0.06$. Since the Hyades cluster is also more metal-rich than the field, with a mean $[\mathrm{Fe} / \mathrm{H}]=+0.14$ (Cayrel de Strobel et al. 1997; Perryman et al. 1998; Grenon 2001), this could also argue in favour of a large proportion of stars in the Hyades stream originating from the Hyades cluster. However, this higher metallicity of the Hyades stream could also indicate that its stars originate from the inner Galaxy.

In this paper, we aim at determining the chemical abundances of stars belonging to the Hyades stream, in order to yield constraints on their origin (from the cluster or from the field of the disc, and in the latter case, from which typical galactocentric radius). In this way we shall be able to independently confirm from chemical tagging that the Hyades stream cannot be solely composed of stars originating in the Hyades cluster, and we shall be able to provide the first direct evidence for two distinct populations inside the stream. We also aim

\footnotetext{
${ }^{1}$ Most likely a 4:1 inner resonance, in order to prevent the corotation from being too far out in the disc.
} 
Table 1. Data for the analysed stellar sample.

\begin{tabular}{|c|c|c|c|c|c|c|c|c|c|}
\hline HD & $\begin{array}{c}d \\
(\mathrm{pc})\end{array}$ & $M_{V}$ & $\begin{array}{c}U \\
\left(\mathrm{~km} \mathrm{~s}^{-1}\right)\end{array}$ & $\begin{array}{c}V \\
\left(\mathrm{~km} \mathrm{~s}^{-1}\right)\end{array}$ & $\begin{array}{c}W \\
\left(\mathrm{~km} \mathrm{~s}^{-1}\right)\end{array}$ & $\begin{array}{l}m_{v} \\
(\mathrm{mag})\end{array}$ & $b-y$ & $\begin{array}{c}\mathcal{M} \\
\left(\mathcal{M}_{\odot}\right)\end{array}$ & $\begin{array}{l}V_{\text {rot }} \sin i \\
\left(\mathrm{~km} \mathrm{~s}^{-1}\right)\end{array}$ \\
\hline \multicolumn{10}{|c|}{ Sure Hyades cluster members } \\
\hline 18632 & 23 & 6.13 & -42 & -19 & -1 & 7.978 & & 0.87 & 3 \\
\hline 19902 & 40 & 5.19 & -41 & -19 & -1 & 8.171 & 0.452 & 0.89 & 2 \\
\hline 26756 & 46 & 5.13 & -41 & -18 & -3 & 8.457 & 0.431 & 0.93 & 5 \\
\hline 26767 & 43 & 4.88 & -41 & -18 & -3 & 8.045 & 0.405 & 0.98 & 5 \\
\hline HIP 13806 & 39 & 5.94 & -41 & -17 & -2 & 8.90 & & 0.89 & 4 \\
\hline \multicolumn{10}{|c|}{ Possible Hyades cluster members } \\
\hline 20430 & 46 & 4.07 & -42 & -23 & -2 & 7.386 & 0.364 & 1.10 & 6 \\
\hline 20439 & 42 & 4.66 & -41 & -21 & -4 & 7.766 & 0.395 & 1.17 & 6 \\
\hline 26257 & 58 & 3.84 & -35 & -13 & -5 & 7.639 & 0.345 & 1.25 & 8 \\
\hline HIP 13600 & 53 & 5.21 & -40 & -13 & -2 & 8.83 & & 1.00 & 2 \\
\hline \multicolumn{10}{|c|}{ Hyades velocity box } \\
\hline 25680 & 17 & 4.79 & -25 & -14 & -6 & 5.903 & 0.399 & 0.96 & 3 \\
\hline 42132 & 111 & 1.52 & -43 & -16 & -23 & 6.675 & 0.525 & 0.89 & 3 \\
\hline 67827 & 45 & 3.39 & -35 & -14 & -9 & 6.580 & 0.368 & 1.34 & 5 \\
\hline 86165 & 51 & 4.39 & -35 & -17 & -16 & 7.926 & 0.386 & 0.97 & 2 \\
\hline 89793 & 63 & 4.87 & -33 & -20 & 12 & 8.853 & 0.424 & 0.96 & 1 \\
\hline 90936 & 56 & 4.62 & -42 & -18 & -14 & 8.371 & 0.389 & 0.96 & 3 \\
\hline 103891 & 58 & 2.79 & -28 & -17 & 7 & 6.591 & 0.356 & 1.35 & 4 \\
\hline 108351 & 88 & 3.19 & -50 & -19 & 5 & 7.905 & 0.327 & 1.40 & 9 \\
\hline 133430 & 57 & 4.79 & -30 & -16 & -5 & 8.559 & 0.413 & 0.90 & 2 \\
\hline 134694 & 134 & 2.87 & -43 & -20 & -17 & 8.502 & 0.336 & 1.34 & 10 \\
\hline 142072 & 39 & 4.90 & -29 & -15 & -1 & 7.854 & 0.417 & 0.90 & 7 \\
\hline 149028 & 48 & 5.10 & -37 & -16 & -1 & 8.530 & 0.454 & 0.91 & 2 \\
\hline 149285 & 196 & 2.36 & -34 & -14 & -14 & 8.825 & 0.337 & 1.32 & 8 \\
\hline 151766 & 109 & 2.42 & -35 & -13 & -20 & 7.606 & 0.348 & 1.46 & 8 \\
\hline 155968 & 55 & 4.72 & -30 & -14 & 2 & 8412 & 0.423 & 0.96 & 3 \\
\hline 157347 & 20 & 4.83 & -28 & -17 & -20 & 6.287 & 0.425 & 0.90 & 1 \\
\hline 162808 & 63 & 4.41 & -31 & -12 & -2 & 8.423 & 0.393 & 1.00 & 6 \\
\hline 171067 & 26 & 5.13 & -46 & -16 & -14 & 7.205 & 0.424 & 0.90 & 1 \\
\hline 180712 & 45 & 4.73 & -25 & -12 & -6 & 7.977 & 0.395 & 0.88 & 3 \\
\hline 187237 & 26 & 4.78 & -36 & -20 & 14 & 6.877 & 0.409 & 0.90 & 2 \\
\hline 189087 & 27 & 5.75 & -42 & -15 & 5 & 7.886 & 0.483 & 0.81 & 2 \\
\hline
\end{tabular}

at making a preliminary analysis of the respective characteristics of these two populations. In Section 2, we present the sample of stars that we are going to analyse, and in Section 3 we briefly describe the observations performed with the High Efficiency and Resolution Mercator Echelle Spectrograph (HERMES) /Mercator spectrograph (Raskin et al. 2011). Stellar parameters and abundances are respectively determined in Sections 4 and 5. We then investigate whether stars in the stream are compatible with being evaporated from the Hyades cluster in Sections 6 and 7, and discuss these results and their consequences in Section 8. Conclusions are drawn in Section 9.

\section{SAMPLE}

Fig. 1 presents the distribution of all stars from the GC survey in the $U-V$ plane ( $U$ is the velocity towards the galactic centre, $V$ the velocity in the direction of Galactic rotation, both with respect to the Sun), and the Hyades overdensity corresponds to the box defined by $-23 \mathrm{~km} \mathrm{~s}^{-1} \leq V \leq-12 \mathrm{~km} \mathrm{~s}^{-1}$ and $-50 \mathrm{~km} \mathrm{~s}^{-1} \leq U \leq-25 \mathrm{~km} \mathrm{~s}^{-1}$ (see Famaey et al. 2007): this box corresponds to a region in which the density of the GC survey in $U V$ velocity space is higher than $\sim 4$ stars $/\left(\mathrm{km} \mathrm{s}^{-1}\right)^{2}$.

A randomly chosen stellar sample within this velocity box has been selected among the GC survey stars with $b-y \geq 0.3$ (or equivalently, $B-V \geq 0.5$, according to tables 15.7 and 15.10 of Drilling \& Landolt 1999) and $V_{\text {rot }} \sin i \leq 10 \mathrm{~km} \mathrm{~s}^{-1}$, the latter condition in order to ease the abundance determination. Members of the Hyades cluster, taken from the De Silva et al. (2006) sample have been added to this GC kinematical sample, in order to compare the element abundances in the stream and in the cluster. The criterion on the colour index of the stream stars is necessary to avoid any bias from the rejection of the fast rotators from the stream: since Paulson, Sneden \& Cochran (2003; their fig. 5) have shown that in the Hyades cluster, all stars (but one) with $B-V \geq 0.5$ have $V_{\text {rot }} \sin i \leq 10 \mathrm{~km} \mathrm{~s}^{-1}$, so that few if any stars evaporated from the cluster have been left aside by selecting only slow rotators with $B-V \geq 0.5$ in the stream.

Among the Hyades cluster members we have selected, four are controversial (see Table 3): they are spatially associated with the cluster but they might not belong to it based on kinematics (de Bruijne, Hoogerwerf \& de Zeeuw 2001).

The full stellar sample is listed in Table 1, the distance and spatial velocities are taken from Holmberg et al. (2009), the masses from Holmberg et al. (2007) and the rotation velocity from Nordström et al. (2004). Positions in the Galaxy and velocities are displayed in Figs 2-5. As each star has been observed individually, the sample is relatively small, comprising 21 stars randomly chosen in the 


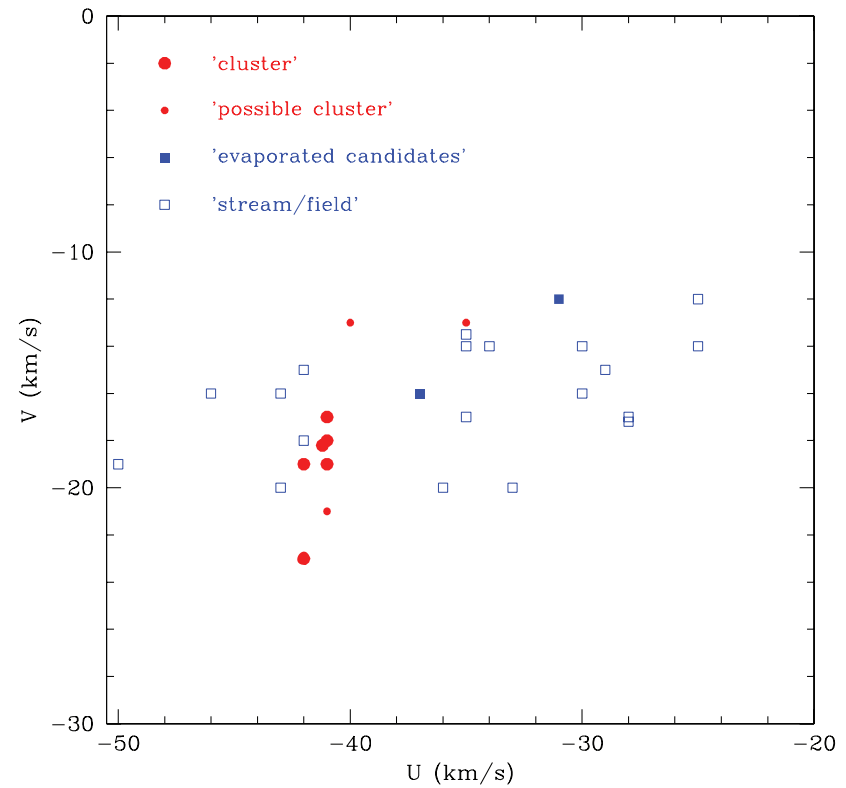

Figure 2. The present stellar sample in the $U-V$ plane. The meaning of the symbols is given in the figure (see Section 6 for more details).

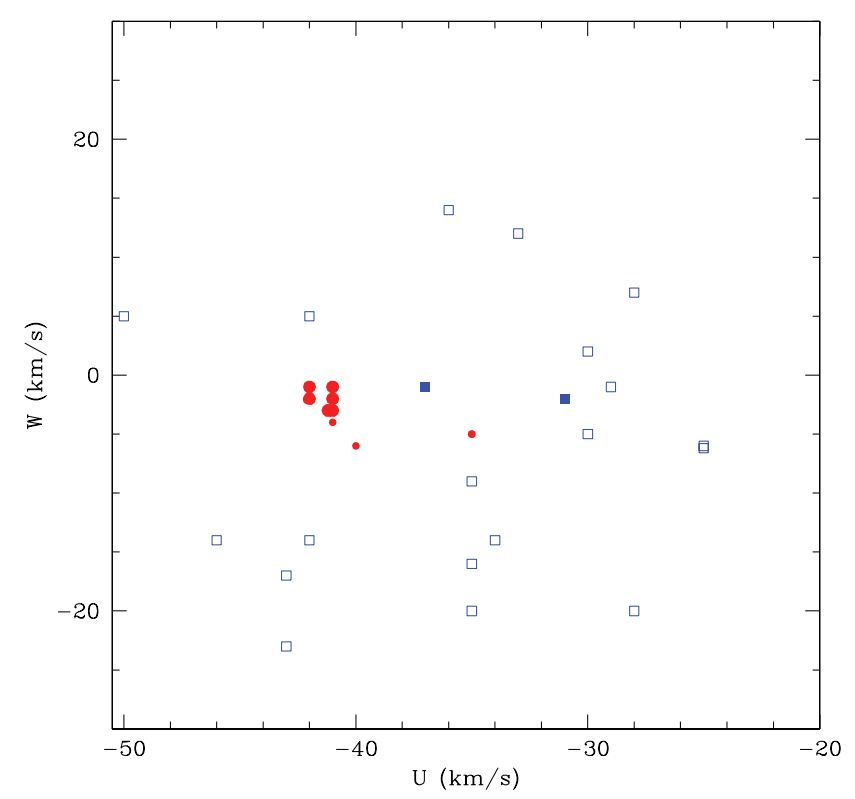

Figure 3. Same as Fig. 2 for the $U-W$ plane.

Hyades box, five sure members of the Hyades cluster and four possible members. This small sample size nevertheless already allows us to draw a number of secure conclusions, as we shall see in the following sections, and paves the way towards more detailed analyses of much larger samples with future multifibre spectrographs such as the High Resolution Multi-Object Spectrograph of the AngloAustralian Telescope, which like the spectrometer used by us also is called HERMES (Barden et al. 2008).

\section{OBSERVATIONS}

The selected stars were observed in 2009 May with the Mercator 1.2-m telescope (Katholieke Universiteit Leuven, Roque de los Muchachos Observatory, La Palma, Spain), using the fiberfed, high-resolution spectrograph HERMES/Mercator (Raskin et al.

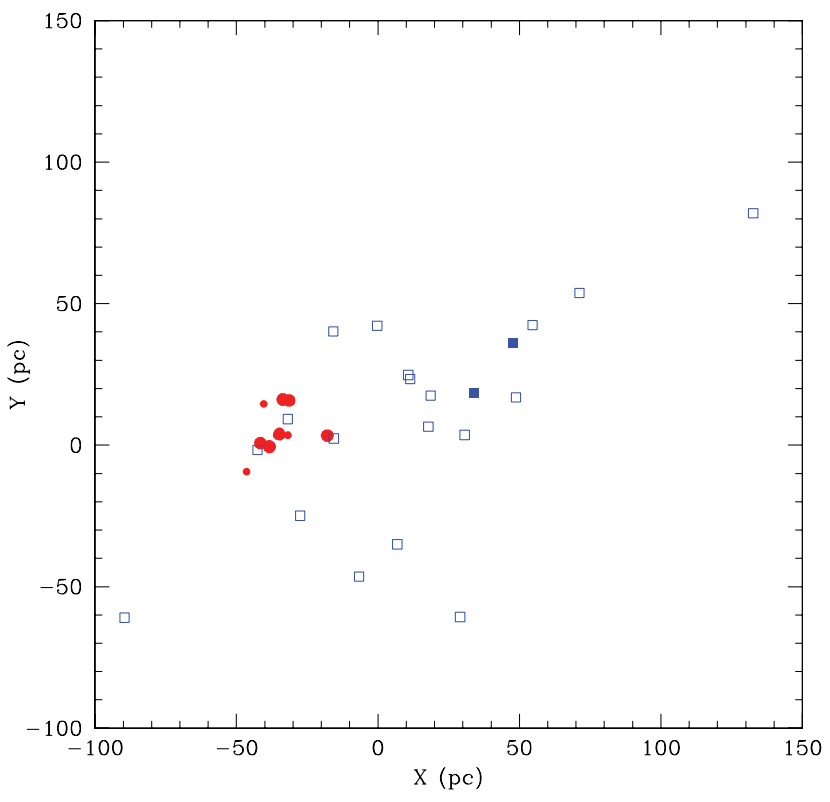

Figure 4. The spatial distribution of Hyades cluster and stream/field stars, projected on the $X-Y$ plane (with $X$ pointing towards the galactic centre and $Y$ in the direction of the galactic rotation; distances on both axes are expressed in parsecs). Symbols are as in Fig. 2.

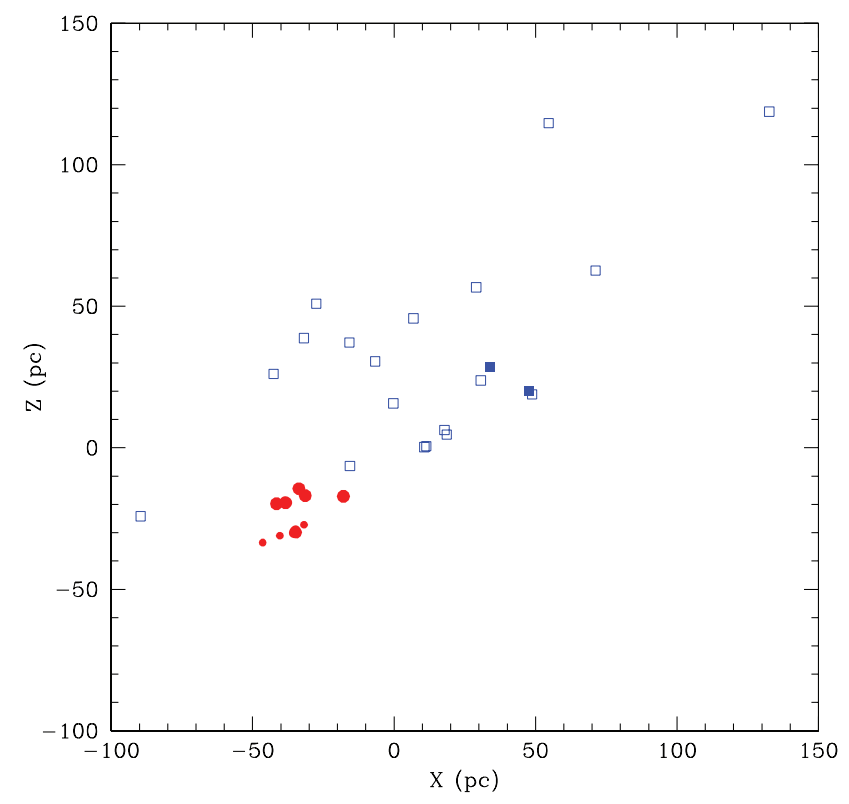

Figure 5. Same as Fig. 4 for the $X-Z$ plane.

2011). The spectra cover the wavelength range 380-900 $\mathrm{nm}$, with a mean resolving power $\lambda / \Delta \lambda=84600$, and a mean signal-to-noise ratio (S/N) of about 125 (the $\mathrm{S} / \mathrm{N}$ for each target star is listed in Table 3). The data were reduced using the automatic pipeline for the HERMES/Mercator spectrograph, complemented by IRAF packages for continuum definition and Doppler correction.

\section{DETERMINATION OF THE STELLAR PARAMETERS}

We have adopted the new Model Atmospheres in Radiative and Convective Scheme (MARCS) model atmospheres (Gustafsson et al. 2008) and interpolated within the grid whenever necessary. The 
Table 2. Line list. An asterisk (*) after the wavelength means that hyperfine structure and isotopic shifts have been taken into account for the considered line. References to the adopted $\log g f$ are given in the last column, and the corresponding reference is listed at the bottom of the table.

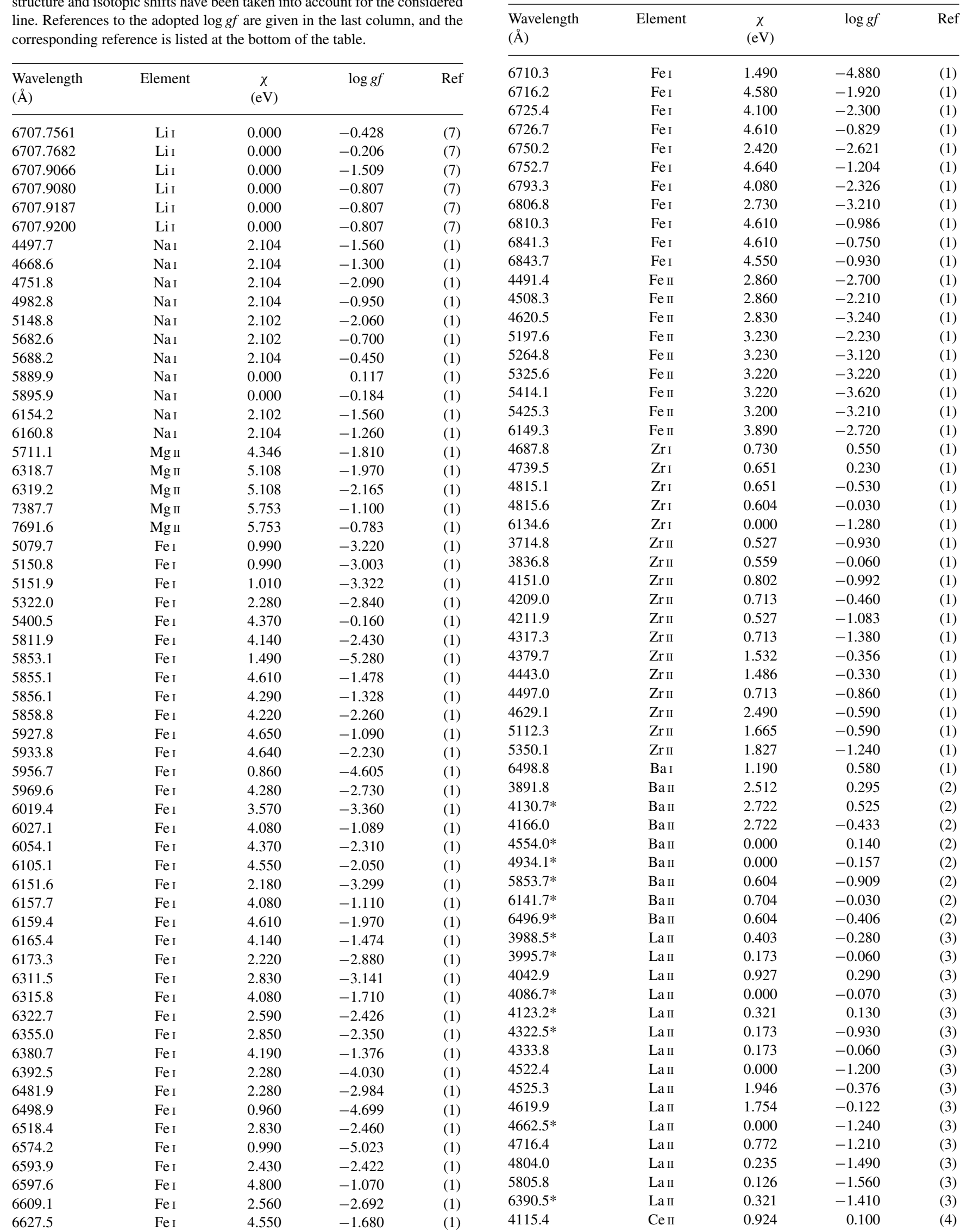

Table 2 - continued 
Table 2 - continued

\begin{tabular}{lcccc}
\hline $\begin{array}{l}\text { Wavelength } \\
(\AA)\end{array}$ & Element & $\begin{array}{c}\chi \\
(\mathrm{eV})\end{array}$ & $\log g f$ & Ref \\
\hline 4137.7 & $\mathrm{Ce}_{\text {II }}$ & 0.516 & 0.440 & $(4)$ \\
4562.4 & $\mathrm{Ce}_{\text {II }}$ & 0.478 & 0.230 & $(4)$ \\
4628.2 & $\mathrm{Ce}_{\text {II }}$ & 0.516 & 0.200 & $(4)$ \\
5274.2 & $\mathrm{Ce}_{\text {II }}$ & 1.044 & 0.150 & $(4)$ \\
4109.5 & $\mathrm{Nd}_{\text {II }}$ & 0.321 & 0.350 & $(5)$ \\
4446.4 & $\mathrm{Nd}$ & 0.205 & -0.350 & $(5)$ \\
5319.8 & $\mathrm{Nd}_{\text {II }}$ & 0.550 & -0.140 & $(5)$ \\
$3724.9^{*}$ & $\mathrm{Eu}_{\mathrm{II}}$ & 0.000 & -0.090 & $(6)$ \\
$4129.7^{*}$ & $\mathrm{Eu}_{\mathrm{II}}$ & 0.000 & 0.220 & $(6)$ \\
$4205.0^{*}$ & $\mathrm{Eu}_{\mathrm{II}}$ & 0.000 & 0.210 & $(6)$ \\
$6049.5^{*}$ & $\mathrm{Eu}_{\mathrm{II}}$ & 1.279 & 0.800 & $(6)$ \\
$6645.1^{*}$ & $\mathrm{Eu}$ II & 1.380 & 0.120 & $(6)$ \\
\hline
\end{tabular}

References: (1) Vienna Atomic Line Database (VALD) (2) Masseron (2006); (3) Lawler, Bonvallet \& Sneden (2001a); (4) Zhang et al. (2001) (Database on Rare Earths at Mons University [DREAM]); (5) Den Hartog et al. (2003); (6) Lawler et al. (2001b); (7) Hobbs, Thorburn \& Rebull (1999).

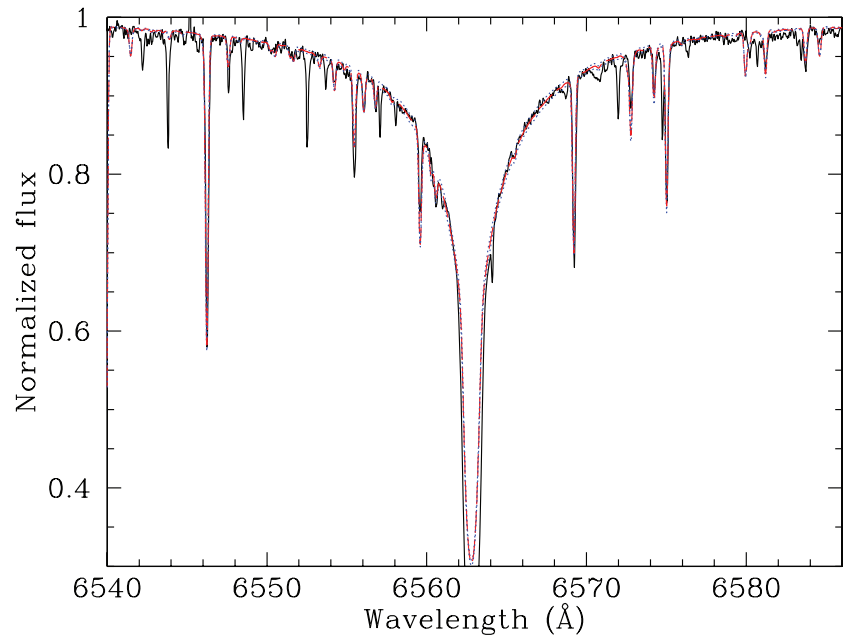

Figure 6. Fit of the $\mathrm{H} \alpha$ line profile of HD 103891, for the adopted $T_{\text {eff }}$ of $5900 \mathrm{~K}$ (red solid line) $\pm 50 \mathrm{~K}$ (blue dotted lines).

stellar parameters were obtained in the following manner. The effective temperature was derived by requiring that the abundances derived from iron lines (as listed in Table 2) exhibit no trend with excitation potential. Gravity was obtained through ionization balance, and microturbulence was set by requiring the absence of a trend between abundances and reduced equivalent widths. It was moreover checked that the wings of the $\mathrm{H} \alpha$ line are correctly reproduced (Fig. 6).

To illustrate the procedure, Fig. 7 displays $[\mathrm{Fe} \mathrm{I} / \mathrm{H}]$ and $[\mathrm{Fe} \mathrm{II} / \mathrm{H}]$ against the line equivalent widths and excitation potentials, for star HD 103891. The difference $T_{\text {eff }}(\mathrm{H} \alpha)-T_{\text {eff }}\left(\mathrm{Fe} \mathrm{I}_{\mathrm{I}}\right)$ averages to $-20 \mathrm{~K}$, with a standard deviation of $33 \mathrm{~K}$ for our complete sample.

The adopted atmospheric parameters are listed in Table 3, where the stars are already grouped according to their abundances and kinematics (the separation procedure will be discussed in Section 6). The metallicity values listed in Table 3 and plotted in Fig. 8 are normalized by the (solar) value $\log \epsilon(\mathrm{Fe})=7.45$ (Asplund, Grevesse $\&$ Sauval 2005). In this table, we also list the $\mathrm{S} / \mathrm{N}$ ratio of the spectra, and the line-to-line abundance scatter $\sigma$. Fig. 8 checks for the absence of any trend between $\left[\mathrm{Fe}_{\mathrm{I}}, \mathrm{Fe}\right.$ II $\left./ \mathrm{H}\right]$ and the atmospheric pa-
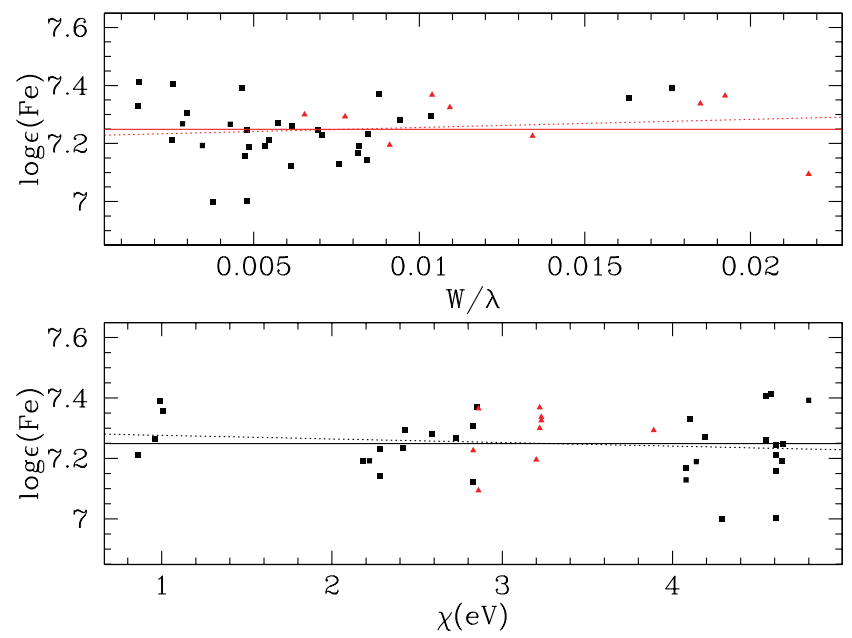

Figure 7. $[\mathrm{Fe} / / \mathrm{H}]$ (black filled squares) and $[\mathrm{Fe}$ II/H] (red filled triangles) against the line equivalent widths and excitation potentials, for star HD 103891. The solid line depicts the adopted abundance, whereas the dotted line is the least-square fit through the data points, with slopes of $2.80 \pm$ 3.27 and $-0.012 \pm 0.014$ for the upper and lower panels respectively, thus consistent with a zero-slope within the error bars.

rameters microturbulence $\xi$, gravity $\log g$ and effective temperature $T_{\text {eff }}$.

The solar metallicity has been derived from the same set of lines as for the program stars, with the following solar-model parameters: $T_{\text {eff }}=5777 \mathrm{~K}, \log g=4.44,[\mathrm{Fe} / \mathrm{H}]=0.00, \xi=1.0 \mathrm{~km} \mathrm{~s}^{-1}$, and using the solar spectrum from Neckel (1999). The solar metallicity $[\mathrm{Fe} / \mathrm{H}]_{\odot}=0.13 \pm 0.12$ listed in Table 3 is just $1 \sigma$ off the expected null value.

For comparison, the atmospheric parameters derived by Paulson et al. (2003) for the stars in common are listed as well in Table 3, along with $T_{\text {eff }}$ from the fit of the $\mathrm{H} \alpha$ wings. For the nine stars in common with Paulson et al. (2003), our final stellar parameters agree well with theirs, since $T_{\text {eff }}$ (Paulson) $-T_{\text {eff }}(\mathrm{Fe}$ I, this work) averages to $70 \mathrm{~K}$ with a standard deviation of $75 \mathrm{~K}$ (and a maximum difference of $200 \mathrm{~K}$ for HD 20430); $\log g$ (Paulson) - $\log g$ (this work) averages to 0.05 with a standard deviation of 0.11 (and a maximum difference of 0.2 dex for HD 20430, HD 20439 and HD 26257). Although our metallicities may appear systematically larger than Paulson et al. ones in Table 3, it must be noted that the normalization value is different, since Paulson et al. (2003) have used $\log \epsilon \odot(H)=7.64$ [as derived from their statement that their value of the solar metallicity differs by 0.14 dex from Grevesse \& Sauval one: $\log \epsilon \odot(H)=$ 7.50]. After allowing for this difference between the normalization values, it turns out that our metallicities are on average 0.08 dex smaller than Paulson ones, the largest difference being 0.14 dex for HD 20439, and the second largest 0.13 dex for HD 20430 and HIP 13806.

\section{ABUNDANCES}

The choice of the elements studied was driven by the finding from previous abundance studies of the Hyades cluster by Paulson et al. (2003) and De Silva et al. (2006) that the star-to-star differential abundance scatter for the elements $\mathrm{Mg}, \mathrm{Zr}, \mathrm{Ba}, \mathrm{La}, \mathrm{Ce}$ and $\mathrm{Nd}$ is especially small (at most $0.055 \mathrm{dex}$ ). To these elements, we have added $\mathrm{Li}, \mathrm{Na}$ and the $r$-process element $\mathrm{Eu}$. Our line list is given in Table 2. Clean lines were first selected on the Sun spectrum. Among those, some were discarded if they were too weak and/or 
Table 3. Stellar parameters. The $[\mathrm{Fe} / \mathrm{H}]$ values from this work are normalized by $\log \epsilon(\mathrm{Fe})=7.45$ (Asplund et al. 2005), while those from Paulson et al. (2003) are normalized by $\log \epsilon(\mathrm{Fe})=7.64$.

\begin{tabular}{|c|c|c|c|c|c|c|c|c|c|c|c|}
\hline HD & $\begin{array}{r}T_{\text {eff }} \\
(\mathrm{K})\end{array}$ & $\log g$ & {$\left[\mathrm{Fe}_{\mathrm{I}} / \mathrm{H}\right] \sigma$} & $n$ & {$[\mathrm{Fe} \mathrm{II} / \mathrm{H}] \sigma$} & $n$ & $\begin{array}{c}\xi \\
\left(\mathrm{km} \mathrm{s}^{-1}\right)\end{array}$ & $\mathrm{S} / \mathrm{N}$ & $\begin{array}{l}T_{\text {eff }} \\
\mathrm{H} \alpha\end{array}$ & $\begin{array}{c}T_{\text {eff }} \quad \log g \quad[\mathrm{Fe} / \mathrm{H}] \\
\quad \text { (Paulson et al. 2003) }\end{array}$ & Membership $^{a}$ \\
\hline
\end{tabular}

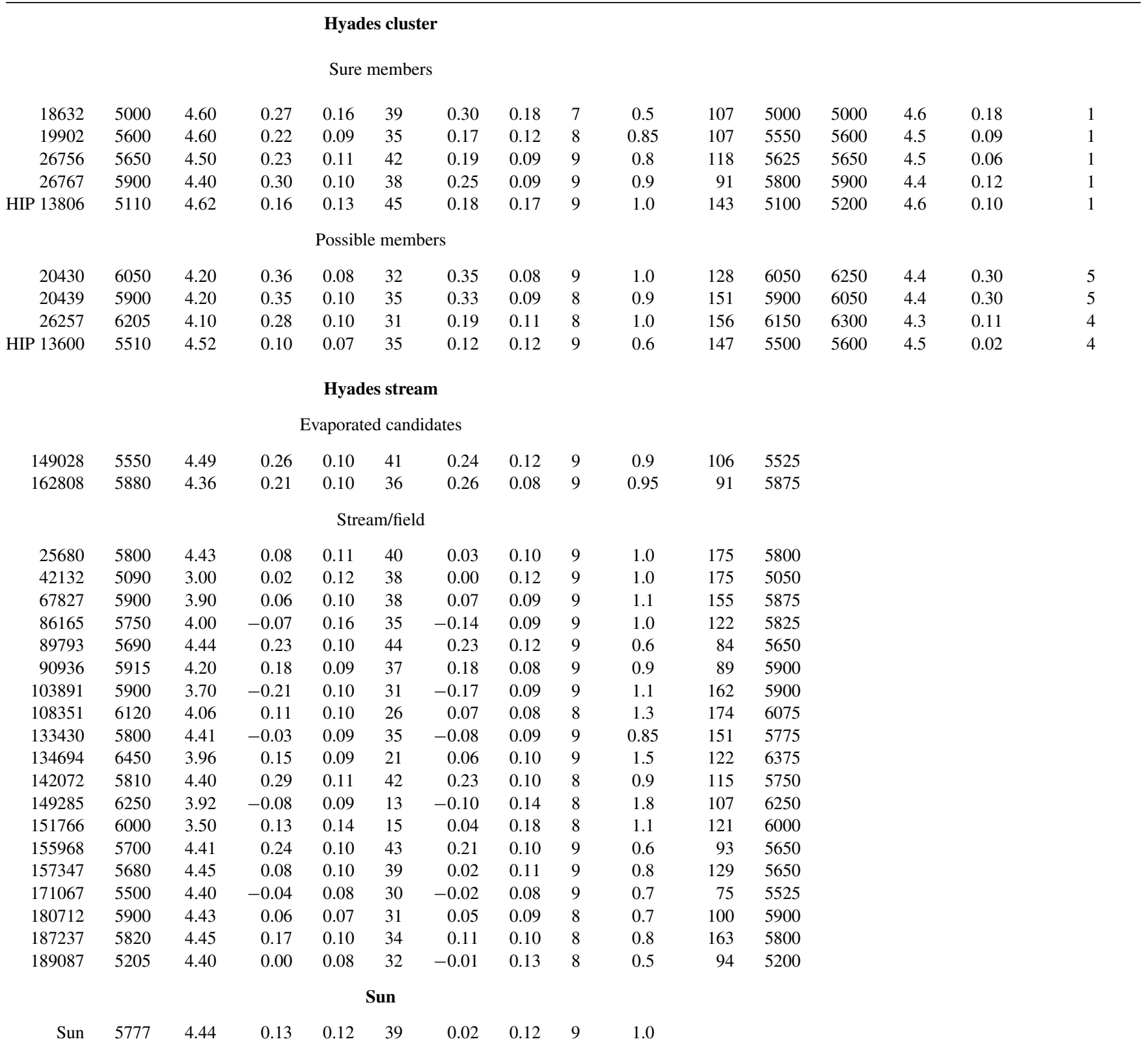

${ }^{a}$ The membership flag is from de Bruijne et al. (2001) according to the following codes: (1) member based on proper motion and radial velocity; (4) member based on proper motion and radial velocity but rejected by Hoogerwerf \& Aguilar (1999); (5) member based on proper motion and radial velocity but rejected by de Bruijne (1999) and Hoogerwerf \& Aguilar (1999).

too severely blended in most of the program stars. Elemental abundances for $\mathrm{Na}, \mathrm{Mg}, \mathrm{Zr}, \mathrm{Ba}, \mathrm{La}, \mathrm{Ce}, \mathrm{Nd}$ and $\mathrm{Eu}$ have been derived from spectral-line synthesis, using the 'TURBOSPECTRUM' package (Alvarez \& Plez 1998), a program devoted to spectrum synthesis in cool stars. The spectral-line-synthesis approach is well suited for complex lines with hyperfine structure splitting (HFS) and/or isotopic shift (IS). The HFS and IS for Ba II have been taken from Masseron (2006), for La II from Lawler et al. (2001a) and for Eu II from Lawler et al. (2001b). Concerning the isotopic composition, we use the solar mixture (Grevesse \& Sauval, 1998), except for Li, for which we neglected the contribution from ${ }^{6} \mathrm{Li}$.

For comparison, solar abundances have been derived using the same line list as for the target stars, with the solar-model parameters listed in Table 3 and using the solar spectrum from Neckel (1999). Solar abundances normalized to $\log \epsilon(\mathrm{H})=12$ are listed in Table 4 .

Errors are expected to come from continuum placement, line fitting and uncertainties in stellar parameters and in atomic data. In order to reduce systematic errors due to the latter, we have performed 

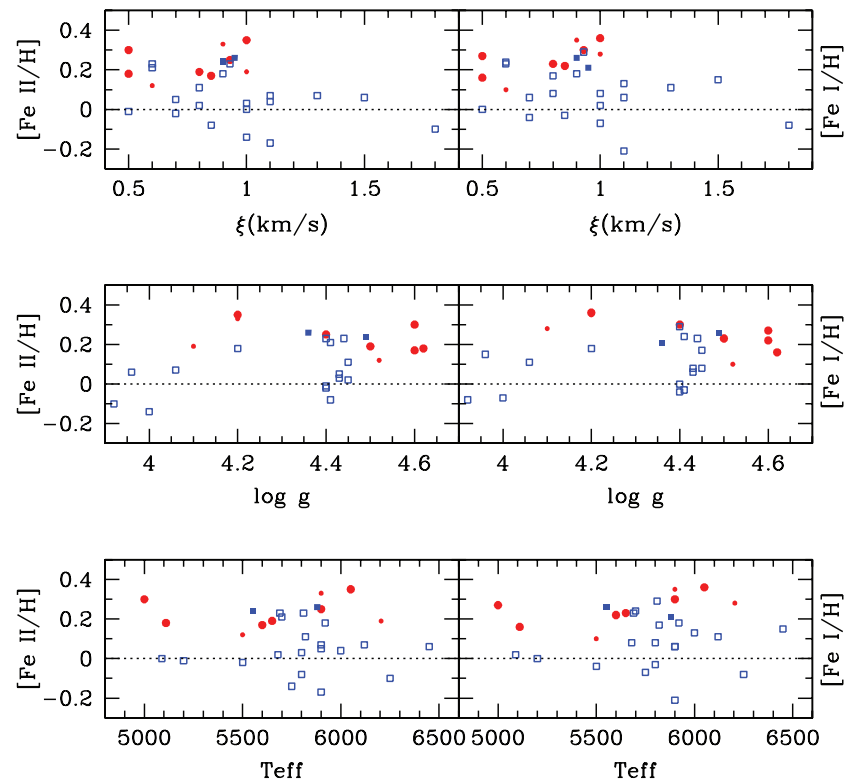

Figure 8. Metallicities as a function of the adopted stellar parameters $T_{\text {eff }}$, $\log g$ and microturbulence $\xi$ (in $\mathrm{km} \mathrm{s}^{-1}$ ). Symbols are as in Fig. 2.

Table 4. Solar elemental abundances, in the scale $\log \epsilon(\mathrm{H})=12$. Column 1 lists the considered ions, column 2 the meteoritic abundances from Grevesse \& Sauval (1998), columns 3 and 4 the solar photospheric standard abundances from Grevesse \& Sauval (1998) and Asplund et al. (2005), respectively. Our solar abundances are given in column 5 .

\begin{tabular}{|c|c|c|c|c|}
\hline \multirow[t]{2}{*}{ Ion } & \multirow[t]{2}{*}{ Meteorites } & \multicolumn{2}{|c|}{ Photosphere } & \multirow[t]{2}{*}{ This study } \\
\hline & & GS98 & As05 & \\
\hline $\mathrm{Na}_{\mathrm{I}}$ & 6.32 & 6.33 & 6.17 & 6.26 \\
\hline Mg II & 7.58 & 7.58 & 7.53 & 7.56 \\
\hline $\mathrm{Fe}_{\mathrm{I}}$ & 7.50 & 7.50 & 7.45 & 7.58 \\
\hline $\mathrm{Fe}_{\text {II }}$ & 7.50 & 7.50 & 7.45 & 7.47 \\
\hline $\mathrm{Zr} \mathrm{I/II}$ & 2.61 & 2.60 & 2.59 & 2.52 \\
\hline Ba II & 2.22 & 2.13 & 2.17 & 2.08 \\
\hline La II & 1.22 & 1.17 & 1.13 & 1.10 \\
\hline $\mathrm{Ce}_{\text {II }}$ & 1.63 & 1.58 & 1.58 & 1.61 \\
\hline $\mathrm{Nd}$ II & 1.49 & 1.50 & 1.45 & 1.38 \\
\hline Eu II & 0.55 & 0.51 & 0.52 & 0.48 \\
\hline
\end{tabular}

a line-by-line differential analysis relative to HD 26756, a member of the Hyades cluster. With this differential approach, errors due to uncertainties in the atomic data $(\log g f)$ are avoided. The differential abundances are given in Table 5 together with the line-to-line scatter $(\sigma)$ and the number of lines used for each element $(n)$. Fig. 9 presents the differential $[\mathrm{X} / \mathrm{Fe}]$ abundances (relative to $\mathrm{HD} 26756$ ) for $\mathrm{Na}$, $\mathrm{Mg}, \mathrm{Zr}, \mathrm{Ba}, \mathrm{La}, \mathrm{Ce}, \mathrm{Nd}$ and Eu against $T_{\text {eff }}$. Except perhaps for $\mathrm{Nd}$ (and to a lesser extent, $\mathrm{Zr}$ ), there is no significant abundance trend with $T_{\text {eff }}$.

Errors due to continuum placement and line blends can be estimated together with random errors from the line-to-line scatter $\sigma$. For most of the lines, the uncertainties due to the line-fitting procedure are less than $\pm 0.02 \mathrm{dex}$, but for a few lines in some stars it can be as high as \pm 0.05 dex. For all elements the reported abundances are from a local thermodynamic equilibrium (LTE) analysis although departures from LTE are expected for $\mathrm{Na}, \mathrm{Mg}, \mathrm{Ba}$ and $\mathrm{Eu}$.

In Table 6, we list the uncertainties associated with the stellar parameters, for two typical stars (HD 149285 and HD 189087).
The adopted uncertainties for the parameters are $\Delta \log g=0.1 \mathrm{dex}$, $\Delta T_{\text {eff }}=50 \mathrm{~K}$ and $\Delta[\mathrm{Fe} / \mathrm{H}]=0.05 \mathrm{dex}$. For the microturbulent velocity, we have adopted $\Delta \xi=0.10 \mathrm{~km} \mathrm{~s}^{-1}$, which is the minimum value for noticeable changes in the slope of $[\mathrm{Fe} / \mathrm{H}]$ (Fig. 7). The total uncertainty $\left(\Delta_{\text {err }}\right)$ has been derived assuming that the errors coming from the uncertainties on the various atmospheric parameters are not correlated (thus taking the square root of the quadratic sum of the different error terms), and is listed in the last column of Table 6 for each element. It turns out that it is Eu which has the largest sensitivity to errors on the stellar-model parameters, since $\Delta_{\text {err }}(\mathrm{Eu})=0.15 \mathrm{dex}$ as compared to less than $0.10 \mathrm{dex}$ for all the other elements.

\section{TAgGing THE POPULATIONS}

The main goal of these abundance measurements is to check whether at least a small fraction of the Hyades stream is compatible with having its origin in the Hyades cluster. To identify possible evaporated stars from the primordial cluster, we computed the $\chi^{2}$ statistics:

$\chi^{2}(1)=\Sigma_{i=\mathrm{Zr}, \mathrm{Ba}, \mathrm{La}, \mathrm{Ce}}\left[\left(\left[X_{i} / \mathrm{Fe}\right]-<\left[X_{i} / \mathrm{Fe}\right]>_{\mathrm{Cluster}}\right) / \sigma_{i, \text { cluster } *}\right]^{2}$,

and

$$
\begin{aligned}
\chi^{2}(2)= & \Sigma_{i=\mathrm{Zr}, \mathrm{Ba}, \mathrm{La}, \mathrm{Ce}}\left[\left(\left[X_{i} / \mathrm{Fe}\right]-<\left[X_{i} / \mathrm{Fe}\right]>_{\text {Cluster }}\right) / \sigma_{i, \text { cluster } *}\right]^{2} \\
& +\left[\left([\mathrm{Fe} \mathrm{I} / \mathrm{H}]-<[\mathrm{Fe} \mathrm{I} / \mathrm{H}]>_{\text {Cluster }}\right) / \sigma_{\mathrm{FeI}, \text { cluster } *}\right]^{2},
\end{aligned}
$$

where the average abundance for the cluster and the star-to-star scatter $\sigma_{\text {cluster* }}$ are taken over the five sure members (see Table 3). Despite the small number of stars involved, the differential abundance scatter used in the above $\chi^{2}$ formulae agree reasonably well with the values derived by Paulson et al. (2003) and De Silva et al. (2006) on a much larger sample of Hyades cluster stars [see $\sigma_{*}$ (DS) and $\sigma_{*}(\mathrm{P} 03)$ in Table 8]. Thus the relatively small number of Hyades cluster stars used in the present study does not appear to hamper our analysis.

The motivation to use two statistical indices $\left[\chi^{2}(1)\right.$ and $\left.\chi^{2}(2)\right]$ based on different elemental sets is to illustrate the sensitivity to specific choices of elemental sets, as we will discuss below. We decided to leave $\mathrm{Li}, \mathrm{Nd}$ and $\mathrm{Eu}$ out of these sets, because

(i) $\mathrm{Nd}$ possibly exhibits an abundance trend with $T_{\text {eff }}$ (see Fig. 9), which could induce a spurious difference between the average $\mathrm{Nd}$ abundances in the stream and cluster, depending on how stars in these two groups distribute with $T_{\text {eff }}$;

(ii) $\mathrm{Eu}$ has the largest sensitivity to errors on the stellar-model parameters (Table 6, where $\Delta_{\text {err }}=0.15$ dex for Eu as compared to less than 0.10 dex for all the other elements);

(iii) $\mathrm{Li}$ is a very sensitive tagger which will be used afterwards (Section 7) as an independent check of the quality of the tagging based on the $\chi^{2}$ statistics from $\mathrm{Fe}$ and the heavier elements.

The different number of degrees of freedom (4 and 5, where we suppose that the average and $\sigma_{*}$ do not act to decrease the number of degrees of freedom; this is certainly true for the stream stars) for the two $\chi^{2}$ statistics makes their direct comparison difficult. It is therefore useful to use the $F 2$ goodness-of-fit statistics instead, which transforms a $\chi^{2}$ statistics into a normal distribution with zero mean and unit variance, irrespective of the number of degrees of freedom. $F 2$ is defined as

$$
F 2=\left(\frac{9 v}{2}\right)^{1 / 2}\left[\left(\frac{\chi^{2}}{v}\right)^{1 / 3}+\frac{2}{9 v}-1\right]
$$




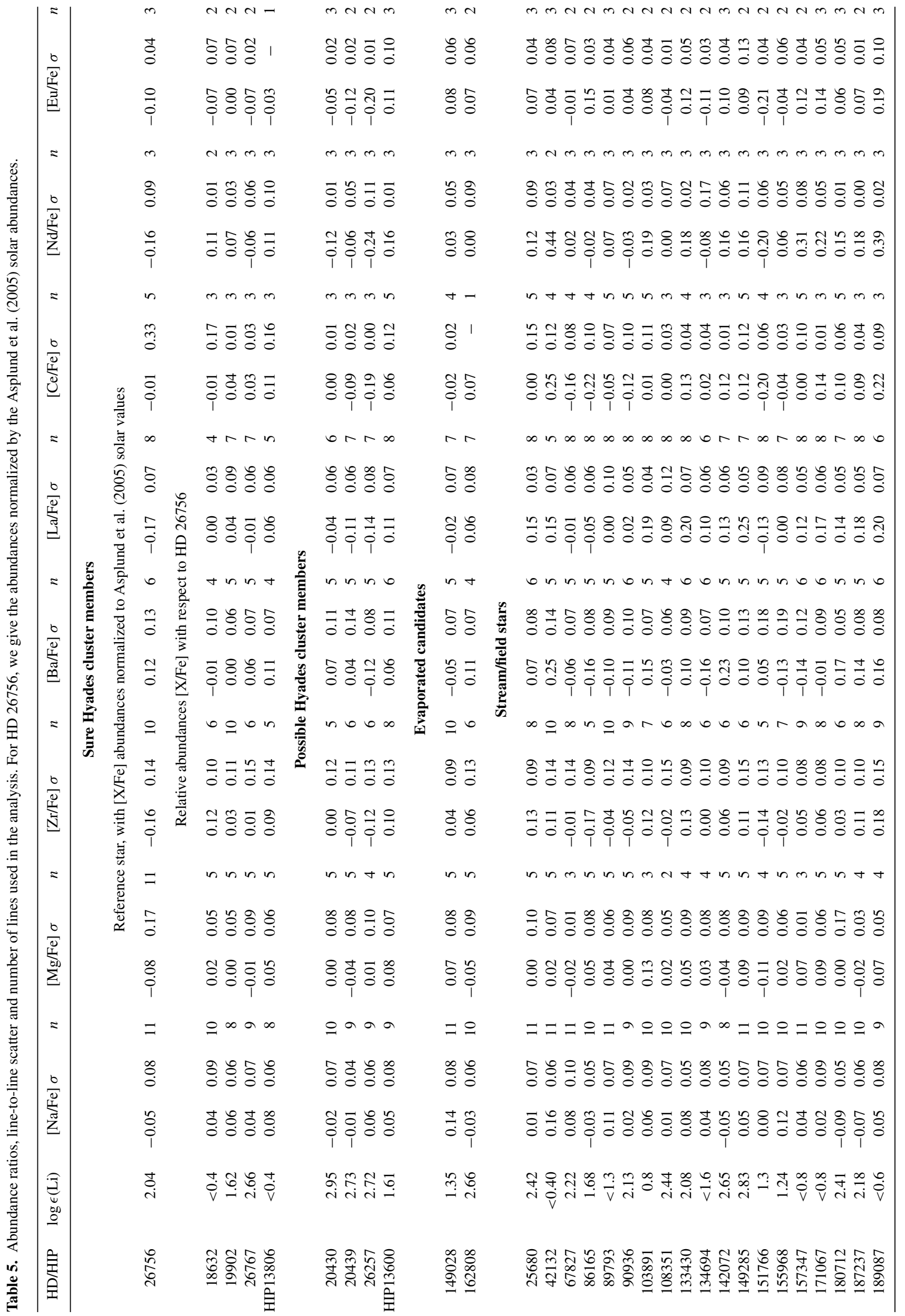



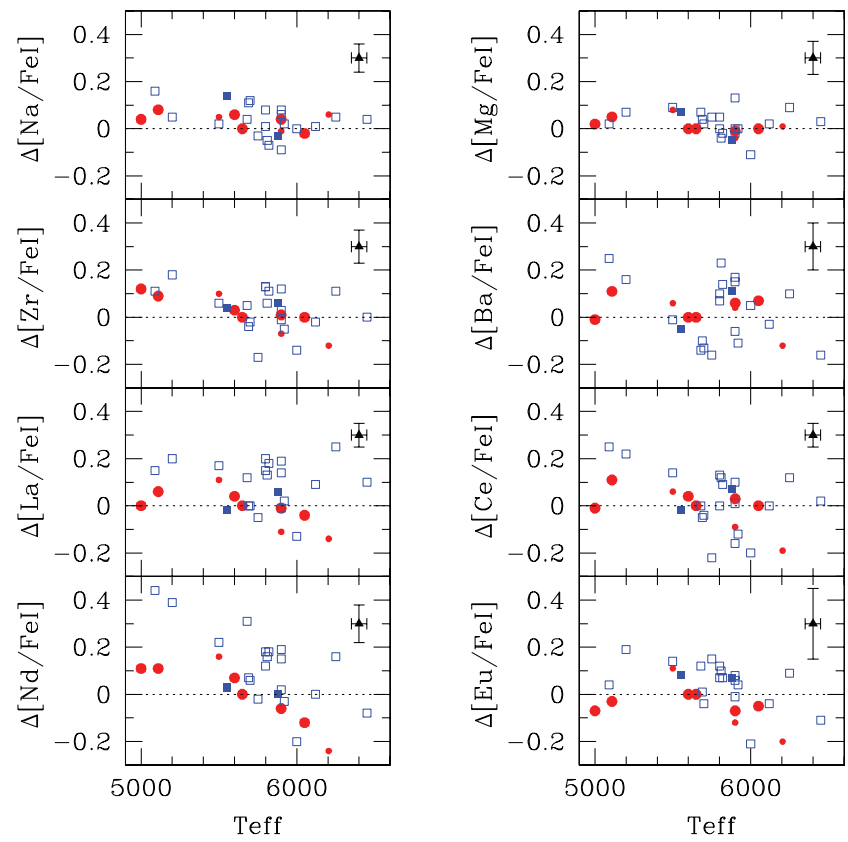

Figure 9. Differential $[\mathrm{X} / \mathrm{Fe}]$ ratios (relative to $\mathrm{HD} 26756$ ) for $\mathrm{Na}, \mathrm{Mg}$, $\mathrm{Zr}, \mathrm{Ba}, \mathrm{La}, \mathrm{Ce}, \mathrm{Nd}$ and Eu against $T_{\text {eff. }}$ Symbols are as in Fig. 2. The error bars drawn in each panel represent the abundance uncertainties due to uncertainties on the stellar parameters (see Table 6). The dashed line corresponds to $[\mathrm{X} / \mathrm{Fe}]=0$.

where $v$ is the number of degrees of freedom of the $\chi^{2}$ variable. The above definition corresponds to the 'cube-root transformation' of the $\chi^{2}$ variable (Stuart \& Ord 1994).

The values of $F 2(1)$ and $F 2(2)$ for all the stars studied are given in Table 7. As apparent from that table, all sure Hyades cluster members (plus the possible member HD 20430) have $F 2<2$. This is actually not really surprising, since the $\sigma_{*}$ value entering the $\chi^{2}$ is derived from the cluster sample itself. The predictive power of this $\chi^{2}$ criterion therefore rather lies in its application to the stream sample. Two stars from the stream (HD 149028 and HD 162808) have both $F 2<2$ and may thus be considered as evaporated from the cluster (the analysis of the Li sequence presented in Section 7 will confirm that conclusion). All other stars, including the possible members (HIP 13600, HD 20439 and HD 26257), have (at least one) $F 2>2$ and must not be considered as cluster members based on the Fe and heavy element abundances, the latter star HD 26257 being even located way off the threshold, with $F 2$ of the order of 7. This star was studied by De Silva et al. (2006) as well, who find relative abundances ${ }^{2} \Delta[\mathrm{Zr} / \mathrm{Fe}]=-0.14, \Delta[\mathrm{Ba} / \mathrm{Fe}]=0.01$, $\Delta[\mathrm{Ce} / \mathrm{Fe}]=-0.20$ and $\Delta[\mathrm{Nd} / \mathrm{Fe}]=-0.05$ not so different form ours: $\Delta[\mathrm{Zr} / \mathrm{Fe}]=-0.12, \Delta[\mathrm{Ba} / \mathrm{Fe}]=-0.12, \Delta[\mathrm{Ce} / \mathrm{Fe}]=-0.19$ and $\Delta[\mathrm{Nd} / \mathrm{Fe}]=-0.24$, the latter set being consistently underabundant, in contrast with the De Silva et al. values. Actually, one may wonder whether the three controversial Hyades cluster stars (HIP 13600, HD 20439 and especially HD 26257) could not in fact be stream stars happening to be located in the spatial vicinity of the cluster. Interestingly, we stress that doubts were raised as well by De Silva et al. (2006) about the membership of HD 20439 (also known as vB 2), based on outlying abundances, in agreement with its outlying $F 2$ obtained in the present study. Incidentally, these au-

${ }^{2}$ The values quoted here are differences from their table 2, with HD 26756 as the reference star. thors raise the same doubts for HD 20430 (vB 1), which our study does not tag as an outlier, however.

In any case, there is a large population of stream stars which are chemically very different from the Hyades cluster members, as apparent from Fig. 10 presenting the distributions of $F 2(1), F 2(2)$ and $\Delta[\mathrm{Fe} / \mathrm{H}]$ for the Hyades stream and cluster. We may thus conclude that, irrespective of the index being used, only a few stars from the Hyades stream have abundances compatible with the abundance ratios of stars of the Hyades cluster (see Section 8.1).

\section{LITHIUM AS AN EFFICIENT POPULATION TAGGER}

We now turn to lithium as a tagger that we use to check the quality of the tagging based on Fe and heavier elements. Boesgaard \& King (2002) have provided a comparison sample of Li abundances in Hyades cluster stars, as shown in Fig. 11 (crosses). The Li abundance in the cluster stars follows a tight sequence that goes through a maximum around $6200 \mathrm{~K}$, and decreases sharply on either side as Li gets destroyed by diffusive or convective downward motions (see Deliyannis 2000 and Sestito \& Randich 2005, for reviews). On the opposite, in field stars, the Li abundance spans a substantial range (of the order of 2 dex) for any given effective temperature (see e.g. Israelian et al. 2009, and references therein), especially on the cooler side of the peak. The tight sequence observed for the Hyades cluster is a direct consequence of the fact that its stars are coeval (Deliyannis 2000). It offers an efficient way to tag stream stars evaporated from the cluster (although one cannot totally exclude the possibility that a stream star falls by chance along the Hyades sequence).

Fig. 11 confirms the chemical tagging analysis performed in Section 6, since the two stars tagged as 'evaporated candidates' indeed lie along the Hyades cluster Li sequence. Conversely, we find three stream stars falling along the Li sequence (HD 25680, HD 142072 and HD 149285), but this can be a chance occurrence, and does not impose a revision of our former tagging (among these three, only HD 25680 has both $F 2<4$ ). Finally, we note that the three 'possible cluster members' nicely fall along the Hyades Li sequence.

\section{DISCUSSION}

\subsection{Can the stream fully originate from the cluster?}

Since Famaey et al. (2007) estimated that the Hyades overdensity in velocity space constitutes $p_{0}=75$ per cent of the Hyades box (this frequency being almost independent of the colour range), we can ask ourselves whether this overdensity can entirely be due to the population of stars evaporated from the Hyades cluster.

However, if this were true, we would expect to have found about 15 (or about 11-19 with Poissonian uncertainties) stars with abundances compatible with the Hyades cluster $\left(p_{0} N=15.75\right.$ with $N=$ 21 ), instead of 2 . This difference leads to a significance level close to $\alpha=1.00$ (within $10^{-9}$ ) for the unilateral rejection of the null hypothesis that all the stars of the velocity overdensity are originating from the Hyades cluster, as obtained from the relation (resulting from the fact that for $p_{0}=0.75$ and $N=21$, the binomial distribution is already well approximated by a Gaussian distribution):

$u_{\mathrm{obs}}=\frac{\left|x-N p_{0}\right|}{\left[N p_{0}\left(1-p_{0}\right)\right]^{1 / 2}} \geq u_{\alpha}$, 
Table 6. Errors in the abundances due to stellar parameter uncertainties $\Delta \log g=0.1 \mathrm{dex}, \Delta T_{\text {eff }}=50 \mathrm{~K}, \Delta[\mathrm{Fe} / \mathrm{H}]=0.05$ $\operatorname{dex}, \Delta \xi=0.10 \mathrm{~km} \mathrm{~s}^{-1} . \Delta_{\text {err }}$ is the total error.

\begin{tabular}{lrrrrrrrrrr}
\hline Element & \multicolumn{4}{c}{ HD 149285 } & \multicolumn{5}{c}{ HD 189087 } \\
& $\Delta T_{\text {eff }}$ & $\Delta \log g$ & $\Delta[\mathrm{Fe} / \mathrm{H}]$ & $\Delta \xi$ & $\Delta_{\text {err }}$ & $\Delta T_{\text {eff }}$ & $\Delta \log g$ & $\Delta[\mathrm{Fe} / \mathrm{H}]$ & $\Delta \xi$ & $\Delta_{\text {err }}$ \\
\hline$\Delta[\mathrm{Na} / \mathrm{Fe}]$ & 0.05 & 0.03 & 0.03 & 0.02 & 0.06 & 0.06 & 0.02 & 0.01 & 0.01 & 0.06 \\
$\Delta[\mathrm{Mg} / \mathrm{Fe}]$ & 0.00 & -0.02 & -0.03 & -0.02 & 0.04 & 0.00 & -0.02 & -0.04 & -0.06 & 0.07 \\
$\Delta[\mathrm{Zr} / \mathrm{Fe}]$ & 0.01 & 0.03 & -0.03 & -0.02 & 0.05 & -0.02 & 0.01 & -0.06 & -0.02 & 0.07 \\
$\Delta[\mathrm{Ba} / \mathrm{Fe}]$ & 0.02 & 0.01 & 0.05 & 0.07 & 0.09 & 0.06 & 0.04 & 0.05 & 0.04 & 0.10 \\
$\Delta[\mathrm{La} / \mathrm{Fe}]$ & 0.02 & 0.03 & 0.02 & 0.00 & 0.04 & 0.02 & 0.02 & 0.05 & 0.00 & 0.05 \\
$\Delta[\mathrm{Ce} / \mathrm{Fe}]$ & 0.02 & 0.03 & 0.04 & 0.03 & 0.06 & 0.03 & 0.03 & 0.03 & 0.01 & 0.05 \\
$\Delta[\mathrm{Nd} / \mathrm{Fe}]$ & 0.02 & 0.02 & 0.06 & 0.04 & 0.07 & 0.03 & 0.04 & 0.06 & 0.02 & 0.08 \\
$\Delta[\mathrm{Eu} / \mathrm{Fe}]$ & -0.07 & -0.04 & -0.12 & -0.08 & 0.16 & -0.05 & -0.06 & -0.11 & -0.07 & 0.15 \\
\hline
\end{tabular}

Table 7. Results from the chemical tagging based on Fe (relative to HD 26756) and the heavy elements (see text for details). Stars are ordered by increasing $F 2$ (2) (see equation 3). The column labelled 'Member' is based on radial-velocity and propermotion criteria (see Table 3 for details). The horizontal line separates, in the cluster columns, the sure Hyades members from the possible members, and in the stream columns, the evaporated members from the stream/field stars.

\begin{tabular}{lccrlrrrr}
\hline Stream & $F 2(1)$ & $F 2(2)$ & {$[\mathrm{Fe}$ I/H $]$} & Cluster & $F 2(1)$ & $F 2(2)$ & {$[\mathrm{Fe}$ I/H $]$} & Member \\
\hline & & & & HD 26767 & -1.22 & -1.24 & 0.08 & 1 \\
& & & & HD 19902 & -0.76 & -1.08 & 0.00 & 1 \\
HD 162808 & 0.90 & 0.65 & -0.02 & HD 20430 & 0.16 & 0.54 & 0.14 & 5 \\
HD 149028 & 0.93 & 0.59 & 0.02 & HD 18632 & 0.50 & 0.16 & 0.04 & 1 \\
& & & & HIP 13806 & 1.82 & 1.99 & -0.09 & 1 \\
HD 108351 & 2.00 & 2.19 & -0.10 & HIP 13600 & 1.94 & 2.48 & -0.14 & 4 \\
HD 89793 & 2.77 & 2.53 & 0.00 & & & & & \\
HD 155968 & 3.22 & 2.97 & 0.01 & & & & & \\
HD 25680 & 3.64 & 3.96 & -0.14 & HD 20439 & 3.99 & 4.00 & & \\
HD 157347 & 3.96 & 4.27 & -0.14 & & & & & \\
HD 90936 & 4.11 & 3.97 & -0.04 & & & & & \\
HD 180712 & 4.12 & 4.53 & -0.16 & & & & & \\
HD 67827 & 4.19 & 4.58 & -0.16 & & & & & \\
HD 134694 & 4.23 & 4.14 & -0.06 & & & & & \\
HD 171067 & 4.55 & 5.50 & -0.25 & & & & & \\
HD 187237 & 4.91 & 4.83 & -0.06 & & & & & \\
HD 142072 & 4.94 & 4.77 & 0.06 & & & & & \\
HD 103891 & 4.99 & 7.27 & -0.42 & & & & & \\
HD 133430 & 5.42 & 6.21 & -0.25 & & & & & \\
HD 149285 & 6.55 & 7.41 & -0.28 & & & & & \\
HD 151766 & 6.69 & 6.65 & -0.06 & & & & & \\
HD 42132 & 7.14 & 7.53 & -0.20 & & & & & \\
HD 189087 & 7.20 & 7.76 & -0.24 & HD 26257 & & & \\
HD 86165 & 7.60 & 8.35 & -0.28 & & & & &
\end{tabular}

where $x=2, u_{\mathrm{obs}}=6.9$ and $u_{\alpha}$ is the abscissa of the reduced normal distribution $\mathcal{N}(0,1)$ such that $\int_{-\infty}^{u_{\alpha}} \mathcal{N}(0,1)=\alpha$.

We are confident that the sample selection criterion $V_{\text {rot }} \sin i \leq$ $10 \mathrm{~km} \mathrm{~s}^{-1}$ (Section 2) does not affect this conclusion, for the following reason: all stream stars of our sample are low-mass mainsequence stars with $b-y \geq 0.3$ (or equivalently $B-V \geq 0.5$, according to tables 15.7 and 15.10 of Drilling \& Landolt 1999), and Paulson et al. (2003) showed that such stars in the Hyades cluster indeed have $V_{\text {rot }} \sin i \leq 10 \mathrm{~km} \mathrm{~s}^{-1}$, so that no candidate evaporated star should have been missed in the stream by rejecting the fast rotators.

This is thus an independent confirmation of the dynamical (most probably resonant) origin of most of the Hyades stream, even though a population of stars evaporated from the Hyades cluster does overlap with this dynamical stream.

\subsection{Properties of the distinct populations of the stream}

Fig. 9 presents the differential abundances $\Delta[\mathrm{X} / \mathrm{Fe}]$ (relative to HD 26756) against $T_{\text {eff }}$ with different symbols identifying the Hyades cluster members (large filled circles), the possible cluster members (small filled circles), evaporated candidates (large filled squares) and finally stars from the stream/field (open squares). In Table 8 , we evaluate the average abundances for each of the elements considered, separately for these four groups. We report the mean and standard deviations $\sigma_{\star}$ for a given chemical element separately for these four samples, and the rms $s_{\text {lines }}$ (taken over all stars of a given group) of the line-to-line scatter $\sigma$ (for a given star, taken from Tables 3 and 5). The $s_{\text {lines }}$ parameter must be seen as an instrumental scatter, to which any extrinsic (physical) scatter $\left(\sigma_{* \text { ext }}\right)$ will add quadratically to yield $\sigma_{\star}$, so that we must expect $\sigma_{\star} \geq s_{\text {lines. }}$. 

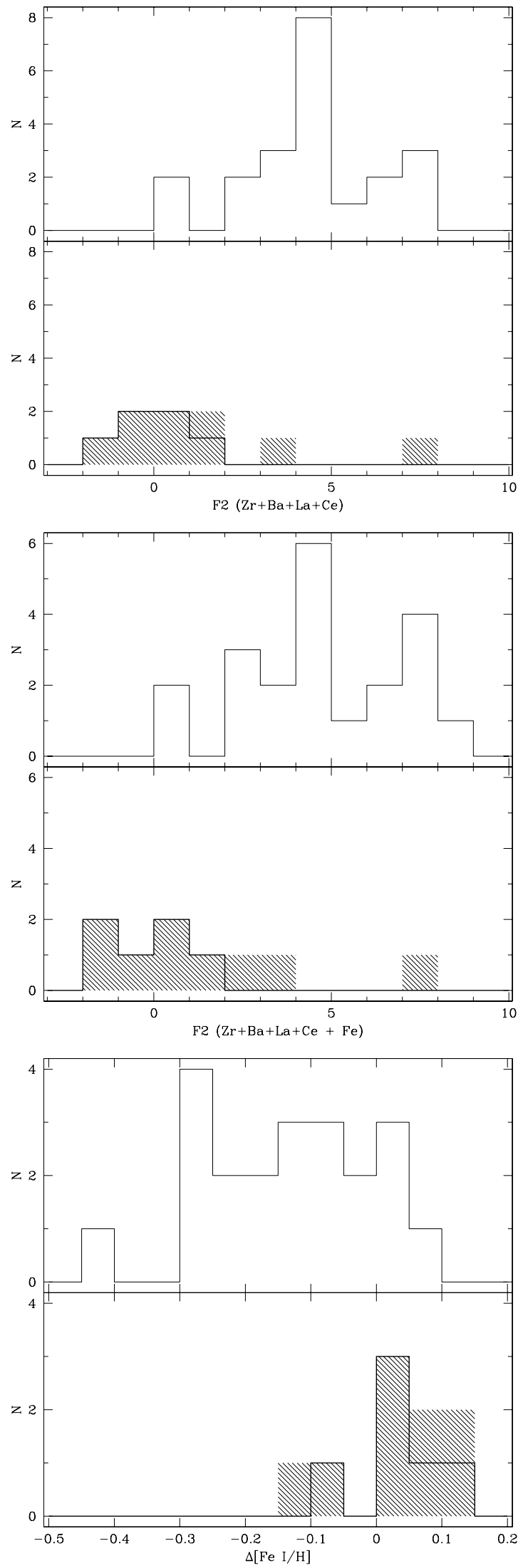

Figure 10. The distributions of $F 2$ (1) (top panel), F2(2) (middle panel) and $\Delta[\mathrm{Fe} / \mathrm{H}]$ (bottom panel) for the Hyades stream stars (open histogram), cluster stars (shaded histogram) and sure cluster stars (thick histogram).

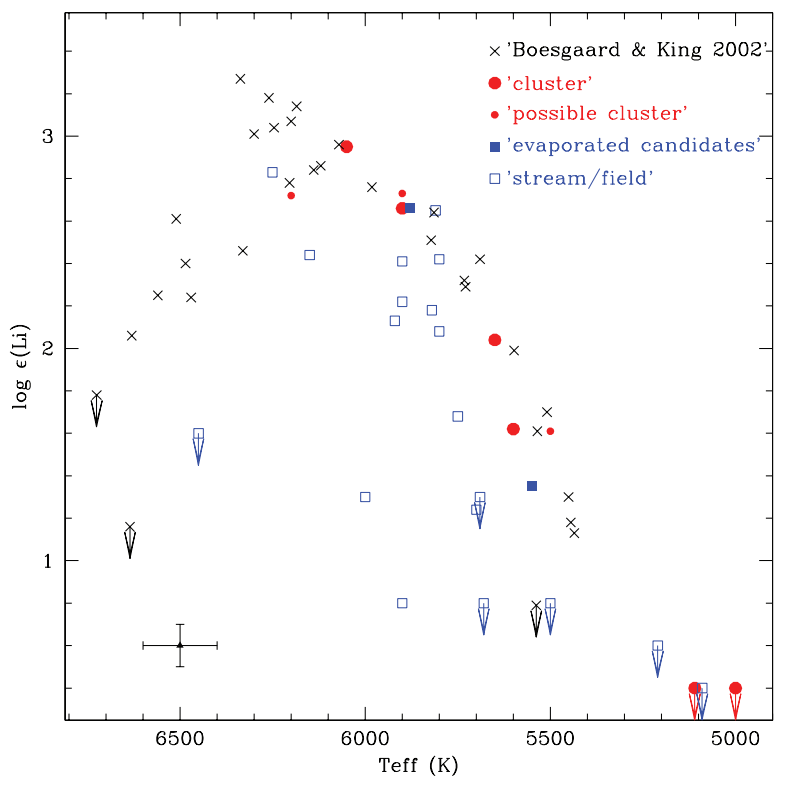

Figure 11. The Li abundance for the different stellar categories as flagged from the $F 2$ indices, compared to Boesgaard \& King (2002) Li abundances for Hyades cluster stars (crosses). Arrows mark upper limits on the $\mathrm{Li}$ abundance.

For the 'cluster' and 'evaporated candidates' samples however, $\sigma_{*}$ is usually smaller than $s_{\text {lines }}$, which indicates that the true scatter of these samples is so small that it cannot be derived accurately with the achieved line-to-line scatter.

A very consistent picture thus emerges from Table 8 regarding the sample genuine scatter $\sigma_{* \text {,ext }}$ : for the cluster sample, it is, as expected, quite small $(\leq 0.05 \mathrm{dex})$ for all elements but $\mathrm{Fe}_{\mathrm{I}}$ and $\mathrm{Nd}$, where it reaches 0.07 dex. Except for these two elements, our results agree well with the conclusion of chemical homogeneity found for 46 Hyades cluster stars by Paulson et al. (2003) and De Silva et al. [2006; compare the entries listed as $\sigma_{*}$ in Table 8 with $\sigma_{*}(\mathrm{DS})$ or $\left.\sigma_{*}(\mathrm{P} 03)\right]$. On the contrary, for the stream, the scatter is systematically much larger, ranging from $0.05 \mathrm{dex}$ for $\mathrm{Mg}$ to $0.15 \mathrm{dex}$ for $\mathrm{Nd}$, as expected if the stream is dominated by field stars. It is not surprising either that the sample of evaporated stars has properties similar to those of the cluster, both in terms of scatter and average value, since they were actually selected to ensure such a similarity. The 'possible cluster' stars deviate markedly from the cluster values, but as indicated in Section 6, the statistical properties of this group are dominated by the outlying star HD 26257. There are as well noteworthy differences (by a least $0.1 \mathrm{dex}$ ) between cluster and stream stars in terms of their average abundances, especially for $\mathrm{Fe}$, $\mathrm{La}, \mathrm{Nd}$ and $\mathrm{Eu}$ (Table 8). The difference in the average metallicity of the two groups (0.2 dex) is very significant (Table 8 and Fig. 10). As apparent from Fig. 12, the difference in the average $\mathrm{La}, \mathrm{Nd}$ and $\mathrm{Eu}$ abundances between the Hyades cluster and stream appears as a natural consequence of their different average metallicities and of the trend with metallicity observed for these elements in the thin disc. This finding is an important result of the present paper: the stream/field population (thus cleaned from the evaporated metalrich cluster stars) remains more metal-rich $([\mathrm{Fe} / \mathrm{H}]$ of the order of $0.04-0.08$, normalized to a solar $\mathrm{Fe}$ abundance of 7.45 , or from -0.01 to 0.05 with the more traditional normalization of 7.50 from Grevesse \& Sauval (2000), in better agreement with older studies; Table 8) than the thin-disc population in the solar neighbourhood. According to Famaey et al. (2007), the mean (photometric) 


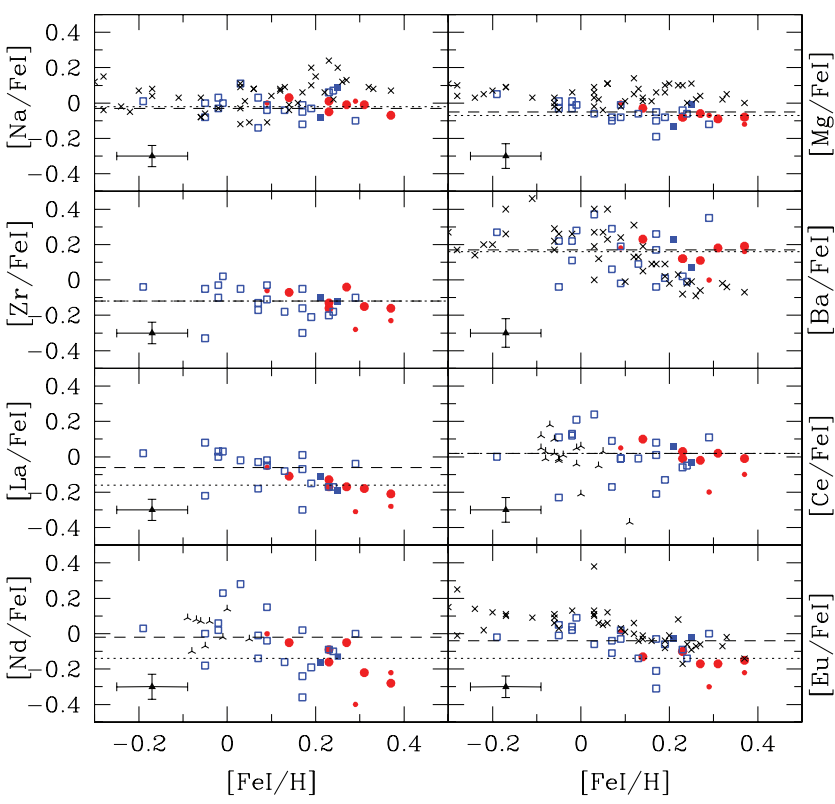

Figure 12. $[\mathrm{X} / \mathrm{Fe}]$ ratios versus $[\mathrm{Fe} / \mathrm{H}]$ for thin-disc stars [four-branch crosses, from Bensby, Feltzing \& Lundström (2003); Bensby et al. (2005) and three-branch crosses from Reddy et al. (2003) for Ce and Nd], certain Hyades cluster members (filled dots), possible Hyades members (open circles), evaporated candidates (filled squares) and stream/field stars (open squares). All abundances in this work are relative to the Asplund et al. (2005) solar abundances. (No attempt has been made to normalize the Bensby et al. and Reddy et al. data in the same way, since their adopted zero-points depend on the spectrograph - see text. Slight offsets between the data sets may thus be expected.) The dotted and dashed lines depict the average cluster and stream abundances, respectively (For $\mathrm{Zr}$ and $\mathrm{Ce}$, they overlap). The error bars drawn in each panel represent the abundance uncertainties due to the line-to-line scatter.

metallicity of the whole GC survey for the solar neighbourhood is $[\mathrm{Fe} / \mathrm{H}]=-0.16$. Excluding halo stars with $[\mathrm{Fe} / \mathrm{H}]<-0.5$, and excluding all the stars from the Hyades box, we get a mean (photometric) metallicity of $[\mathrm{Fe} / \mathrm{H}]=-0.12$. Given that photometric metallicity estimates are systematically smaller than spectroscopic values by about 0.05 dex (Holmberg et al. 2009), the thin-disc metallicity in the solar neighbourhood may be evaluated at $-0.07 \mathrm{dex}$ (see also Allende-Prieto 2010, and references therein). Comparing this thin-disc metallicity of $-0.07 \mathrm{dex}$ with that of the stream/field (of the order of -0.01 to $0.08 \mathrm{dex}$ ) clearly supports an inner-disc origin for the stream. We stress that this comparison is somewhat biased by the fact that about 25 per cent of the stars in the Hyades velocity box must belong to the local background Schwarzschild velocity ellipsoid (Section 1 and Famaey et al. 2007), and thus have a metallicity typical of the thin disc. One may try to clean the sample from the local background field stars to better evaluate the metallicity of the stream stars. An upper limit to the average stream metallicity may be obtained by removing from the 21 stars making up our Hyades box the 25 per cent most metal-deficient stars, believed to belong to the field. Thus removing the most metal-poor stars HD 86165, HD 103891, HD 133430, HD 149285 and HD 171067 from the stream/field sample, one finds an average metallicity of $0.114 \mathrm{dex}$ (with the $\log \epsilon=7.45$ normalization), corresponding to an excess of (at most) 0.18 dex with respect to the metallicity of the local thin disc.

The argument of an inner-disc origin for the stream may even be made more quantitative by adopting a reasonable metallicity gradient of -0.05 to $-0.07 \mathrm{dex} \mathrm{kpc}^{-1}$ in the galactic disc (e.g. Daflon \& Cunha 2004): this would indicate that the Hyades stream originates at $\sim 0.9-3.0 \mathrm{kpc}$ (or even at $3.6 \mathrm{kpc}$ if one adopts the upper limit to the metallicity excess, or even more taking into account the fact that the gradient for old stars may be even flatter) inwards in the Galactic disc. In Section 8.3, this range will be compared with predictions made in the framework of a stream caused by an inner 4:1 resonance from a two-armed spiral pattern (inner ultra-harmonic resonance or IUHR).

The large difference in the average metallicities of the Hyades cluster and stream (after cleaning the latter from the two evaporated candidates) definitely and undoubtedly confirms that the stream is not solely made out of evaporated cluster stars. And concomitantly, this finding reinforces the hypothesis of the dynamical nature of the Hyades stream. In this context, one may thus compare the scatter and abundance trends obtained for the Hyades stream stars with those in the inner thin disc from which they originate. This is done in Fig. 12 using the samples of Bensby et al. $(2003,2005)$ and Reddy et al. (2003). It is seen that the stream conforms with the properties of the thin disc: absence of metallicity trend and small scatter for $\mathrm{Mg}$, relatively large scatter for $\mathrm{Ba}$, and small scatter and well-marked trend for Eu (Fig. 12). The small shifts between our values and Bensby's ones, apparent for $\mathrm{Mg}$ and $\mathrm{Eu}$, may be attributed to the slightly different line list and solar abundances adopted for normalization, with $\log \epsilon$ of $7.53,7.45$ and 0.52 adopted in this work for $\mathrm{Mg}, \mathrm{Fe}$, and $\mathrm{Eu}$ respectively, as compared to 7.57-7.58, 7.53-7.56 and 0.47-0.56 for Bensby et al. (2005; their table 5, where the adopted normalization abundances may depend upon the spectrograph and line used).

\subsection{A resonance from the spiral pattern?}

The question now is which dynamical effects in the Galaxy can both place the Hyades stream in its observed location in velocity space, while also having a metallicity typical of stars originating at $0.9-3.0 \mathrm{kpc}$ inside the solar circle.

As was shown by Minchev et al. (2010), a resonance with the Galactic bar cannot account for the position of the Hyades stream in the $U-V$ plane (while it actually can account for the Pleiades), nor for an origin farther away than $\sim 0.8 \mathrm{kpc}$ in the inner disc. On the other hand, using an orbital weighting function technique, Quillen \& Minchev (2005) showed that the 4:1 IUHR of a two-armed spiral structure $^{3}$ splits the velocity distribution into two features corresponding to two orbital families, one of them consistent with the Hyades stream (see also Section 1). ${ }^{4}$ To check this possibility, we performed test-particle simulations of a stellar disc consistent with the Milky Way kinematics, perturbed by a two-armed spiral pattern. Details about the simulation parameters can be found in Minchev \& Quillen (2007). We examined a range of pattern speeds $\Omega_{\mathrm{s}}$, and indeed found that we could reproduce the position of the Hyades stream in the $U-V$ plane (using the solar motion of Schönrich, Binney \& Dehnen 2010) only when the solar circle is near the $4: 1$ IUHR. Fig. 13 presents our results for $\Omega_{\mathrm{s}}=18 \mathrm{~km} \mathrm{~s}^{-1} \mathrm{kpc}^{-1}$ for an angular speed at the solar position $\Omega_{0}=28 \mathrm{~km} \mathrm{~s}^{-1} \mathrm{kpc}^{-1}$ (i.e. $\left.\Omega_{\mathrm{s}} / \Omega_{0}=0.65\right)$. The top panel shows the two orbital families and the bottom panel the resulting velocity distribution in the $U-V$ plane.

\footnotetext{
${ }^{3}$ Observations indicate that the Milky Way has a four-armed structure, but with two more prominent arms (see e.g. Englmaier, Pohl \& Bissantz 2008). ${ }^{4}$ Note that this splitting into different orbital families of different average galactic radii is also seen at the 4:1 inner Lindblad resonance of a four-armed pattern (see e.g. Amaral \& Lépine 1997).
} 
Table 8. For each group (cluster, evaporated candidates, possible cluster and stream/field, with the membership criterion based on F2; see Section 6) and each chemical element considered, the table lists the mean ( \pm error on the mean), standard deviation $\sigma_{*}$ and $\mathrm{rms} s_{\text {lines }}$ of the line-to-line scatter. The differential abundance scatter $\sigma_{*}(\mathrm{P} 03)$ and $\sigma_{*}(\mathrm{DS})$ obtained by Paulson et al. (2003) and De Silva et al. (2006; their table 5), respectively, from a differential abundance analysis of an extensive sample of 46 Hyades stars are listed as well for comparison. The true scatter of the sample $\left(\sigma_{* \text { ext }}\right)$ is obtained from $\left(\sigma_{*}^{2}-s_{\text {lines }}^{2}\right)^{1 / 2}$. All abundances are differential with respect to HD 26756. The last column lists the $[\mathrm{X} / \mathrm{Fe} \mathrm{I}]$ ( or [ $[\mathrm{Fe} \mathrm{I}, \mathrm{II} / \mathrm{H}]$ ) abundances of the reference star HD 26756, relative to the Asplund et al. (2005) solar abundances.

\begin{tabular}{|c|c|c|c|c|c|}
\hline & Cluster & $\begin{array}{l}\text { Evaporated } \\
\text { candidates }\end{array}$ & $\begin{array}{l}\text { Possible } \\
\text { cluster }\end{array}$ & $\begin{array}{l}\text { Stream/field } \\
\text { population }\end{array}$ & $\begin{array}{l}\text { HD } 26756 \text { relative to solar } \\
\text { (Asplund et al. 2005) }\end{array}$ \\
\hline$N$ & 6 & 2 & 3 & 19 & \\
\hline$F 2$ & $<2$ & $<2$ & $\geq 2$ & $\geq 2$ & \\
\hline$\langle\Delta[\mathrm{Na} / \mathrm{Fe}]\rangle$ & $0.03 \pm 0.01$ & $0.05 \pm 0.06$ & $0.03 \pm 0.02$ & $0.03 \pm 0.01$ & -0.05 \\
\hline$\sigma_{*}(\Delta[\mathrm{Na} / \mathrm{Fe}])$ & 0.032 & 0.09 & 0.03 & 0.06 & \\
\hline$\sigma_{*}(\mathrm{P} 03)$ & 0.06 & & & & \\
\hline$s_{\text {lines }}(\Delta[\mathrm{Na} / \mathrm{Fe}])$ & 0.06 & 0.07 & 0.06 & 0.07 & \\
\hline$\sigma_{*, \mathrm{ext}}$ & $<0.03$ & 0.05 & $<0.03$ & $<0.06$ & \\
\hline$\langle\Delta[\mathrm{Mg} / \mathrm{Fe}]\rangle$ & $0.01 \pm 0.01$ & $0.01 \pm 0.04$ & $0.02 \pm 0.03$ & $0.03 \pm 0.01$ & -0.08 \\
\hline$\sigma_{*}(\Delta[\mathrm{Mg} / \mathrm{Fe}])$ & 0.021 & 0.06 & 0.05 & 0.05 & \\
\hline$\sigma_{*}(\mathrm{P} 03)$ & 0.04 & & & & \\
\hline$s_{\text {lines }}(\Delta[\mathrm{Mg} / \mathrm{Fe}])$ & 0.06 & 0.09 & 0.09 & 0.08 & \\
\hline$\sigma_{*, \mathrm{ext}}$ & $<0.02$ & $<0.06$ & $<0.05$ & $<0.05$ & \\
\hline$\langle\Delta[\mathrm{Fe} / \mathrm{H}]\rangle$ & $0.03 \pm 0.03$ & $0.00 \pm 0.01$ & $0.02 \pm 0.07$ & $-0.14 \pm 0.03$ & 0.23 \\
\hline$\sigma_{*}\left(\Delta\left[\mathrm{Fe}_{\mathrm{I}} / \mathrm{H}\right]\right)$ & 0.072 & 0.02 & 0.12 & 0.12 & \\
\hline$\sigma_{*}(\mathrm{P} 03)$ & 0.05 & & & & \\
\hline$s_{\text {lines }}\left(\Delta\left[\mathrm{Fe}_{\mathrm{I}} / \mathrm{H}\right]\right)$ & 0.08 & 0.06 & 0.06 & 0.09 & \\
\hline$\sigma_{*, \mathrm{ext}}$ & $<0.07$ & $<0.02$ & 0.10 & 0.08 & \\
\hline$\langle\Delta[\mathrm{Fe}$ II $/ \mathrm{H}]\rangle$ & $0.05 \pm 0.03$ & $0.06 \pm 0.01$ & $0.03 \pm 0.05$ & $-0.15 \pm 0.03$ & 0.19 \\
\hline$\sigma_{*}(\Delta[\mathrm{Fe} \mathrm{II} / \mathrm{H}])$ & 0.068 & 0.01 & 0.09 & 0.11 & \\
\hline$s_{\text {lines }}(\Delta[\mathrm{Fe}$ II/H] $)$ & 0.07 & 0.08 & 0.06 & 0.08 & \\
\hline$\sigma_{*, \text { ext }}$ & 0.00 & $<0.01$ & 0.07 & 0.07 & \\
\hline$\langle\Delta[\mathrm{Zr} / \mathrm{Fe}]\rangle$ & $0.04 \pm 0.02$ & $0.05 \pm 0.01$ & $-0.03 \pm 0.05$ & $0.03 \pm 0.02$ & -0.16 \\
\hline$\sigma_{*}(\Delta[\mathrm{Zr} / \mathrm{Fe}])$ & 0.046 & 0.01 & 0.09 & 0.09 & \\
\hline$\sigma_{*}(\mathrm{DS})$ & 0.055 & & & & \\
\hline$s_{\text {lines }}(\Delta[\mathrm{Zr} / \mathrm{Fe}])$ & 0.06 & 0.07 & 0.06 & 0.11 & \\
\hline$\sigma_{*, \text { ext }}$ & $<0.05$ & $<0.01$ & 0.07 & $<0.09$ & \\
\hline$\langle\Delta[\mathrm{Ba} / \mathrm{Fe}]\rangle$ & $0.04 \pm 0.02$ & $0.03 \pm 0.06$ & $-0.01 \pm 0.05$ & $0.03 \pm 0.03$ & 0.12 \\
\hline$\sigma_{*}(\Delta[\mathrm{Ba} / \mathrm{Fe}])$ & 0.044 & 0.08 & 0.08 & 0.13 & \\
\hline$\sigma_{*}(\mathrm{DS})$ & 0.049 & & & & \\
\hline$s_{\text {lines }}(\Delta[\mathrm{Ba} / \mathrm{Fe}])$ & 0.08 & 0.07 & 0.11 & 0.10 & \\
\hline$\sigma_{*, \mathrm{ext}}$ & $<0.04$ & 0.04 & $<0.08$ & 0.08 & \\
\hline$\langle\Delta[\mathrm{La} / \mathrm{Fe}]\rangle$ & $0.01 \pm 0.01$ & $0.02 \pm 0.03$ & $-0.04 \pm 0.06$ & $0.10 \pm 0.02$ & -0.17 \\
\hline$\sigma_{*}(\Delta[\mathrm{La} / \mathrm{Fe}])$ & 0.033 & 0.04 & 0.11 & 0.10 & \\
\hline$\sigma_{*}(\mathrm{DS})$ & 0.025 & & & & \\
\hline$s_{\text {lines }}(\Delta[\mathrm{La} / \mathrm{Fe}])$ & 0.06 & 0.08 & 0.07 & 0.07 & \\
\hline$\sigma_{*, \text { ext }}$ & $<0.03$ & $<0.04$ & 0.08 & 0.07 & \\
\hline$\langle\Delta[\mathrm{Ce} / \mathrm{Fe}]\rangle$ & $0.03 \pm 0.02$ & $0.02 \pm 0.03$ & $-0.08 \pm 0.06$ & $0.02 \pm 0.03$ & -0.01 \\
\hline$\sigma_{*}(\Delta[\mathrm{Ce} / \mathrm{Fe}])$ & 0.041 & 0.04 & 0.10 & 0.13 & \\
\hline$\sigma_{*}(\mathrm{DS})$ & 0.025 & & & & \\
\hline$s_{\text {lines }}(\Delta[\mathrm{Ce} / \mathrm{Fe}])$ & 0.10 & 0.02 & 0.07 & 0.08 & \\
\hline$\sigma_{*, \mathrm{ext}}$ & $<0.04$ & 0.04 & 0.08 & 0.10 & \\
\hline$\langle\Delta[\mathrm{Nd} / \mathrm{Fe}]\rangle$ & $0.02 \pm 0.04$ & $0.01 \pm 0.01$ & $-0.05 \pm 0.09$ & $0.12 \pm 0.04$ & -0.16 \\
\hline$\sigma_{*}(\Delta[\mathrm{Nd} / \mathrm{Fe}])$ & 0.087 & 0.02 & 0.16 & 0.15 & \\
\hline$\sigma_{*}(\mathrm{DS})$ & 0.032 & & & & \\
\hline$s_{\text {lines }}(\Delta[\mathrm{Nd} / \mathrm{Fe}])$ & 0.05 & 0.07 & 0.07 & 0.07 & \\
\hline$\sigma_{*, \text { ext }}$ & 0.07 & $<0.02$ & 0.15 & 0.14 & \\
\hline
\end{tabular}


Table 8 - continued

\begin{tabular}{llllll}
\hline & Cluster & $\begin{array}{l}\text { Evaporated } \\
\text { candidates }\end{array}$ & $\begin{array}{l}\text { Possible } \\
\text { cluster }\end{array}$ & $\begin{array}{l}\text { Stream/field } \\
\text { population }\end{array}$ & $\begin{array}{l}\text { HD 26756 relative to solar } \\
\text { (Asplund et al. 2005) }\end{array}$ \\
\hline$N$ & 6 & 2 & 3 & 19 & \\
$F 2$ & $<2$ & $<2$ & $\geq 2$ & $\geq 2$ & \\
$\langle\Delta[\mathrm{Eu} / \mathrm{Fe}]\rangle$ & $-0.04 \pm 0.01$ & $0.07 \pm 0.01$ & $-0.07 \pm 0.07$ & $0.05 \pm 0.02$ & -0.10 \\
$\sigma_{*}(\Delta[\mathrm{Eu} / \mathrm{Fe}])$ & 0.028 & 0.01 & 0.13 & 0.09 & \\
$S_{\text {lines }}(\Delta[\mathrm{Eu} / \mathrm{Fe}])$ & 0.06 & 0.06 & 0.06 & 0.06 & \\
$\sigma *$, ext & 0.03 & $<0.01$ & 0.11 & 0.07 & \\
\hline
\end{tabular}
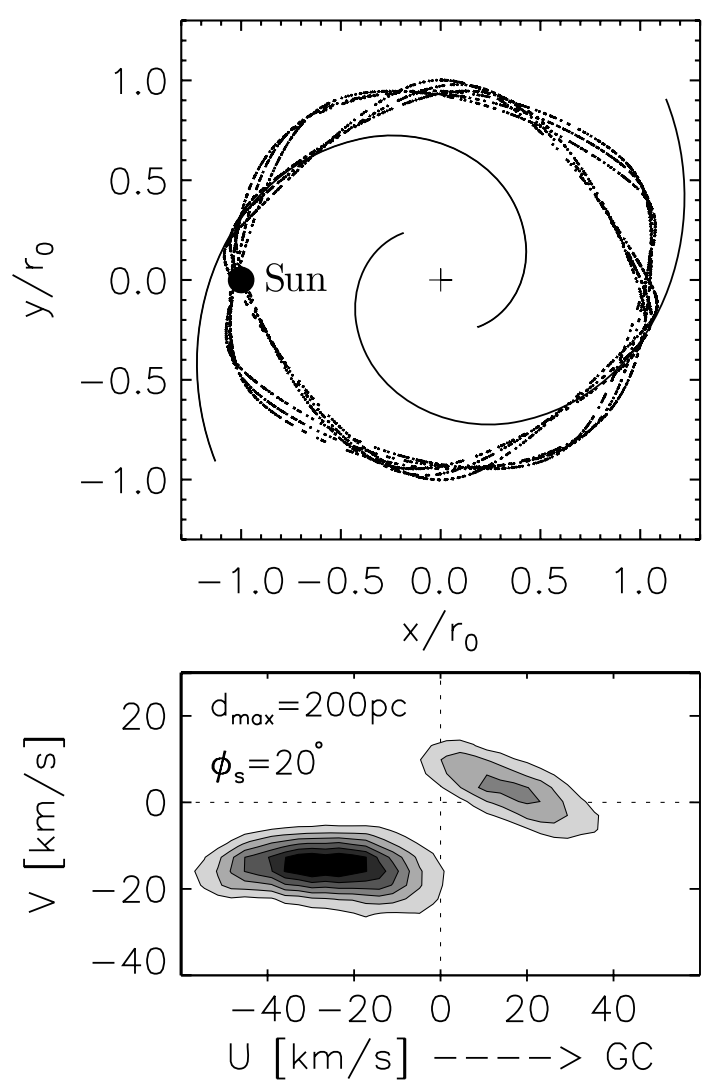

Figure 13. Top panel: the effect of a two-armed spiral structure on orbits near the 4:1 IUHR. Note the splitting into two families of closed orbits in the frame moving with the (trailing) spiral pattern. For a Sun orientation at $20^{\circ}$ with respect to a concave arm, both orbital families enter the solar neighbourhood stellar velocity distribution (black filled circle). The galactocentric axes are in units of $r_{0}$ (the galactocentric radius of the Sun). Bottom panel: the effect on the $U-V$ plane for the configuration shown in the top panel (selecting test particles in a $200 \mathrm{pc}$ circle around the Sun). Each orbital family gives rise to a stream in velocity space. We can associate the dense clump at $(U, V) \approx(-35,-17) \mathrm{km} \mathrm{s}^{-1}$ with the Hyades and the shallow one at $(U, V) \approx(10,0) \mathrm{km} \mathrm{s}^{-1}$ with Sirius. The contour levels correspond to $0.2,0.31,0.43,0.55,0.67$ and 0.8 of the maximum value at the centre of the Hyades clump.

The beauty of this simulation is that, while reproducing the Hyades stream, the other orbital family creates another remarkable feature in velocity space around $(U, V) \approx(10,0) \mathrm{km} \mathrm{s}^{-1}$, which is consistent with the Sirius moving group (see e.g. Famaey et al. 2005, 2008). It is slightly shallower than the Hyades stream, exactly as observed. This simulation furthermore predicts that the Hyades stream originates about $1 \mathrm{kpc}$ inward from the solar radius, which lies within the error bar derived in Section 8.2 from the observed metallicity difference (local thin disc - Hyades stream: $0.06-0.15 \mathrm{dex})$ and metallicity gradient in the thin disc $(-0.05$ to $-0.07 \mathrm{dex} \mathrm{kpc}^{-1}$; Daflon \& Cunha 2004). This error bar is unfortunately quite large still $(0.9-3.0 \mathrm{kpc})$ to meaningfully constrain the simulation, and one should strive to reduce it in the future. If in the end, a metallicity difference as large as (or larger than) 0.1 dex were confirmed, agreement with the present simulation could only be restored at the expense of the necessity for a steeper metallicity gradient in the disc. Let us however note that Daflon \& Cunha (2004) studied OB stars, i.e. the very recent metallicity gradient, and that the gradient for older stars such as those of the Hyades stream is expected to be flatter due to radial mixing, not steeper. This might cast doubt on the ability of the present dynamical model to reproduce the observed metallicity excess, should it be confirmed by further studies. On the other hand, there exists other mechanisms that could have made the Hyades stream migrate from larger distances in the inner disc. It is known that stars can exchange angular momentum in galactic discs due to three distinct mechanisms: transient spirals (Sellwood \& Binney 2002), spiral-bar resonance overlap (Minchev $\&$ Famaey 2010) and the effect of minor mergers on the disc (Quillen et al. 2009). One of these could be responsible for migrating the Hyades stream from a distance larger than what is possible with a spiral structure model only. The 'migrating' stream could remain coherent for a long enough time before dispersing, but more work is needed to investigate this possibility. The drawback would then be that the Hyades and Sirius overdensities in the $U-V$ plane would be unrelated and would need two separate explanations.

\section{CONCLUSION}

From an analysis of the $\mathrm{Li}, \mathrm{Na}, \mathrm{Mg}, \mathrm{Zr}, \mathrm{Ba}, \mathrm{La}, \mathrm{Ce}, \mathrm{Nd}$ and $\mathrm{Eu}$ abundances of 21 stars from the Hyades stream, we performed chemical tagging to identify stream stars possibly evaporated from the cluster. The first tagging method is based on a $\chi^{2}$ test comparing the abundances of $\mathrm{Fe}, \mathrm{Zr}, \mathrm{Ba}, \mathrm{La}$ and $\mathrm{Ce}$ in stream stars with the cluster average value and internal scatter. It is convenient to make use of the 'cube-root transformation' of the $\chi^{2}$ variable to convert it into the goodness-of-fit statistics $F 2$, which behaves as a normal distribution with zero mean and unit variance. This method relies of course on an accurate evaluation of the cluster average value and internal scatter. This tagging method works best for chemical elements exhibiting a steep slope with metallicity. Any metallicity difference between the stream and the cluster is then amplified by each element involved in the $\chi^{2}$ sum and exhibiting a steep metallicity trend. The absence of any such metallicity trend among $\mathrm{Na}$ and $\mathrm{Mg}$ (at least for metallicities in the range -0.1-0.3) makes them useless for chemical tagging. A second method uses the tight $\mathrm{Li}-T_{\text {eff }}$ sequence observed among Hyades stars, a result of the slow $\mathrm{Li}$ destruction process at work in these stars. Stars of the same $T_{\text {eff }}$ 
but of a different age, as will be the case in general for stream stars, will not fall along the Hyades sequence. This method offers the advantage that the range spanned by $\mathrm{Li}$ abundances is very large (of the order of $3 \mathrm{dex}$ ), but is restricted to stars in the narrow $T_{\text {eff }}$ range $5000-6500 \mathrm{~K}$. Outside this range, the $\mathrm{Li}$ abundance is either too small to be measurable or not sensitive to age any longer. Based on these methods, only two stars from the stream (HD 149028 and HD 162808) appear to have abundances within ' $2 \sigma$ ' of the cluster (i.e. $F 2<2$, and matching the $\mathrm{Li}$ sequence); they thus very likely evaporated from the cluster.

Our analysis thus convincingly demonstrates that a large fraction of stars in the Hyades velocity box ( $\sim 90$ per cent) cannot originate from the Hyades cluster. Interestingly, two evaporated candidates have been found, grossly in line with the conclusion of Famaey et al. (2007) that about 15 per cent of the Hyades stream could still originate in the Hyades cluster. Coincidentally, a fully simultaneous analysis of an independent sample of 26 stream stars (De Silva et al. 2011) reached the same conclusion, finding 4 evaporated candidates. It is then of high interest to clean the stream population from this evaporated population and to compare its properties with the local thin disc. It is found here that these stream stars are more metal-rich than the local thin disc, with an excess of the order of $0.06-0.15 \mathrm{dex}$. This metallicity excess implies an origin for the stream at about $0.9-3.0 \mathrm{kpc}$ inwards from the Sun, adopting a galactic metallicity gradient of -0.05 to $-0.07 \mathrm{dex} \mathrm{kpc}^{-1}$. Predictions from a 4:1 inner resonance of a two-armed spiral pattern locate the origin of the Hyades stream at a maximum of $1 \mathrm{kpc}$ inwards from the solar radius. A more precise determination of the stream metallicity is thus needed to confirm or contradict this scenario. Besides reproducing the Hyades overdensity in the $U-V$ plane, this scenario however makes another appealing prediction, namely the existence of the Sirius moving group exactly as observed.

As a conclusion, the analysis of this small subsample of 21 stars from the Hyades stream has already yielded some interesting insights on its nature, and it will be of prime importance to confirm these with much larger samples, e.g. in future analyses performed with multifibre spectrographs such as the High Resolution MultiObject Spectrograph of the Anglo-Australian Telescope (Barden et al. 2008). If they could derive a more accurate metallicity for the stream cleaned from the evaporated population, these large surveys could clearly provide a meaningful constraint on the distance at which the Hyades stream originates, and on the metallicity gradient in the disc, thus shedding light on the dynamical process responsible for the formation of the stream. This, together with the mapping of large-scale non-axisymmetric motions (see e.g. Siebert et al. 2011) will help better constraining the non-axisymmetry of the Galactic potential and its influence on the dynamical and chemical evolution of the disc.

\section{ACKNOWLEDGMENTS}

The HERMES/Mercator project is a collaboration between the K.U. Leuven, the Université libre de Bruxelles and the Royal Observatory of Belgium with contributions from the Observatoire de Genève (Switzerland) and the Thüringer Landessternwarte Tautenburg (Germany). It is funded by the Fund for Scientific Research of Flanders (FWO) under the grant G.0472.04, from the Research Council of K.U. Leuven under grant GST-B4443, from the Fonds National de la Recherche Scientifique (F.R.S.-FNRS) under contracts IISN4.4506.05 and FRFC 2.4533.09, and financial support from Lotto (2004) assigned to the Royal Observatory of Belgium. LP acknowledges financial support from the Département des Re- lations Internationales of the Université libre de Bruxelles and thanks the F.R.S-FNRS (Belgium) for granting her a Bourse de séjour scientifique. Support has also been provided by the Actions de recherche concertées (ARC) (Heavy Elements in the Universe: Stellar Evolution, Nucleosynthesis and Abundance Determinations) from the Direction générale de l'Enseignement non obligatoire et de la Recherche scientifique - Direction de la Recherche scientifique - Communauté française de Belgique. BF acknowledges support from the CNRS (France) and AvH foundation (Germany). GG is a post-doctoral researcher of the FWO-Vlaanderen (Belgium). SVE is FNRS research associate. Authors contributions: LP, TM, SVE, AJ and CSn have conducted the spectroscopic analysis; BF, AJ, IM, AS, JRDL and OB have conducted the dynamical and statistical analyses; CSi, GG, TD and EP have conducted the observations; SVE, AJ, HVW, CW, GR, SP, WP, HH, YF and LD are builders of the HERMES/Mercator spectrograph.

\section{REFERENCES}

Allende Prieto C., 2010, in Cunha K., Spite M., Barbuy B., IAU Symp. 265, Chemical Abundances in the Universe: Connecting First Stars to Planets. Cambridge Univ. Press, Cambridge, p. 304

Alvarez R., Plez B., 1998, A\&A, 330, 1109

Amaral L. H., Lépine J. R. D., 1997, MNRAS, 286, 885

Antoja T., Valenzuela O., Pichardo B., Moreno E., Figueras F., Fernández D., 2009, ApJ, 700, L78

Asplund M., Grevesse N., Sauval A. J., 2005, in Barnes T. G. III, Bash F. N., eds, ASP Conf. Ser. Vol. 336, Cosmic Abundances as Records of Stellar Evolution and Nucleosynthesis. Astron. Soc. Pac., San Francisco, p. 25

Baranne A., Mayor M., Poncet J. L., 1979, Vistas Astron., 23, 279

Barden S. C., Bland-Hawthorn J., Churilov V. et al., 2008, in McLean I. S., Casali M. M., eds, Proc. SPIE, Vol. 7014, Ground-based and Airborne Instrumentation for Astronomy II, 70144J.

Bensby T., Feltzing S., Lundström I., 2003, A\&A, 410, 527

Bensby T., Feltzing S., Lundström I., Ilyin I., 2005, A\&A, 433, 185

Bensby T., Oey M. S., Feltzing S., Gustafsson B., 2007, ApJ, 655, L89

Bland-Hawthorn J., Krumholz M. R., Freeman K., 2010, ApJ, 713, 166

Boesgaard A. M., King J. R., 2002, ApJ, 565, 587

Bovy J., Hogg D. W., 2010, ApJ, 717, 617

Cayrel de Strobel G., Soubiran C., Friel E. D., Ralite N., Francois P., 1997, A\&AS, 124, 299

Daflon S., Cunha K., 2004, ApJ, 617, 1115

de Bruijne J. H. J., 1999, MNRAS, 306, 381

de Bruijne J. H. J., Hoogerwerf R., de Zeeuw P. T., 2001, A\&A, 367, 111

De Silva G. M., Sneden C., Paulson D. B., Asplund M., Bland-Hawthorn J., Bessell M. S., Freeman K. C., 2006, AJ, 131, 455

De Silva G. M., Freeman K. C., Bland-Hawthorn J., Asplund M., Bessell M. S., 2007, AJ, 133, 694

De Silva G. M., Freeman K. C., Bland-Hawthorn J., 2009, Publ. Astron. Soc. Australia, 26, 11

De Silva G. M., Freeman K. C., Bland-Hawthorn J., Asplund M., Williams M., Holmberg J., 2011, MNRAS, in press (arXiv:1103.2588)

Dehnen W., 1998, AJ, 115, 2384

Deliyannis C. P., 2000, in Pallavicini R., Micela G., Sciortino S., eds, ASP Conf. Ser. Vol. 198, Stellar Clusters and Associations: Convection, Rotation, and Dynamos. Astron. Soc. Pac., San Francisco, p. 235

Den Hartog E. A., Lawler J. E., Sneden C., Cowan J. J., 2003, ApJS, 148, 543

Drilling J. S., Landolt A. U., 1999, in Cox A. N., ed., Allen's Astrophysical Quantities, 4th edn. Springer, New York

Eggen O. J., 1958, MNRAS, 118, 65

Eggen O. J., 1978, ApJ, 222, 203

Englmaier P., Pohl M., Bissantz N., 2008, in Corsini E. M., Debattista V. P., eds, Tumbling, Twisting, and Winding Galaxies: Pattern Speeds along the Hubble Sequence. (arXiv0812.3491E) 


\section{4}

\section{Pompéia et al.}

Famaey B., Jorissen A., Luri X., Mayor M., Udry S., Dejonghe H., Turon C., 2005, A\&A, 430, 165

Famaey B., Pont F., Luri X., Udry S., Mayor M., Jorissen A., 2007, A\&A, 461, 957

Famaey B., Siebert A., Jorissen A., 2008, A\&A, 483, 453

Feltzing S., Holmberg J., 2000, A\&A, 357, 153

Freeman K., Bland-Hawthorn J., 2002, ARA\&A, 40, 487

Fux R., 2001, A\&A, 373, 511

Gonzalez G., 2006, PASP, 118, 1494

Grenon M., 2001, in Garcia Lopez R. J., Rebolo R., Zapaterio Osorio M. R., eds, Stellar Systems and the Sun, 11th Cambridge Workshop on Cool Stars. Astron. Soc. Pac., San Fancisco, p. 359

Grevesse N., Sauval A. J., 1998, Space Sci. Rev., 85, 161

Grevesse N., Sauval A. J., 2000, in Manuel O., ed., Origin of Elements in the Solar System, Implications of Post-1957 Observations. Kluwer, Dordrecht, p. 261

Gustafsson B., Edvardsson B., Eriksson K., Jørgensen U. G., Nordlund Å, Plez B., 2008, A\&A, 486, 951

Hobbs L. M., Thorburn J. A., Rebull L. M., 1999, ApJ, 523, 797

Holmberg J., Nordström B., Andersen J., 2007, A\&A, 475, 519

Holmberg J., Nordström B., Andersen J., 2009, A\&A, 501, 941

Hoogerwerf R., Aguilar L. A., 1999, MNRAS, 306, 394

Israelian G. et al., 2009, Nat, 462, 189

Lawler J. E., Bonvallet G., Sneden C., 2001a, ApJ, 556, 452

Lawler J. E., Wickliffe M. E., den Hartog E. A., Sneden C., 2001b, ApJ, 563,1075

Masseron T., 2006, PhD thesis, Observatoire de Paris, France
Minchev I., Famaey B., 2010, ApJ, 722, 112

Minchev I., Quillen A. C., 2007, MNRAS, 377, 1163

Minchev I., Boily C., Siebert A., Bienayme O., 2010, MNRAS, 407, 2122

Neckel H., 1999, Sol. Phys., 184, 421

Nordström B., Mayor M., Andersen J. et al., 2004, A\&A, 418, 989

Paulson D. B., Sneden C., Cochran W. D., 2003, AJ, 125, 3185

Perryman M. A. C. et al., 1998, A\&A, 331, 81

Quillen A. C., 2003, AJ, 125, 785

Quillen A. C., Minchev I., 2005, AJ, 130, 576

Quillen A. C., Minchev I., Bland-Hawthorn J., Haywood M., 2009, MNRAS, 397,1599

Raskin G. et al., 2011, A\&A, 526, A69

Reddy B. E., Tomkin J., Lambert D. L., Allende Prieto C., 2003, MNRAS, 340, 304

Schönrich R., Binney J., Dehnen W., 2010, MNRAS, 403, 1829

Sellwood J., 2010, MNRAS, 409, 145

Sellwood J., Binney J., 2002, MNRAS, 336, 785

Sestito P., Randich S., 2005, A\&A, 442, 615

Siebert A. et al., 2011, MNRAS, 412, 2026

Stuart A., Ord J. K., 1994, in Stuart A., Ord J. K., eds, Kendall's Advanced Theory of Statistics. Vol.1: Distribution Theory. Arnold Publ., London Woolley R., 1961, Obser., 81, 203

Zhang Z. G., Svanberg S., Jiang Z., Palmeri P., Quinet P., Quinet P., Biémont E., Biémont E., 2001, Phys. Scr., 63, 122

This paper has been typeset from a $\mathrm{T}_{\mathrm{E}} \mathrm{X} / \mathrm{L} \mathrm{T}_{\mathrm{E}} \mathrm{X}$ file prepared by the author. 